ARTICLE

DOI: $10.1038 /$ ncomms13324 OPEN

\title{
Saponin-based adjuvants induce cross-presentation in dendritic cells by intracellular lipid body formation
}

Martijn H. den Brok ${ }^{1,2}$, Christian Büll1, Melissa Wassink ${ }^{1}$ Annemarie M. de Graaf ${ }^{1}$, Jori A. Wagenaars ${ }^{1}$, Marthe Minderman ${ }^{1}$, Mayank Thakur ${ }^{3}$, Sebastian Amigorena ${ }^{4}$, Eric O. Rijke ${ }^{5}$, Carla C. Schrier ${ }^{5}$

$\&$ Gosse J. Adema ${ }^{1}$

Saponin-based adjuvants (SBAs) are being used in animal and human (cancer) vaccines, as they induce protective cellular immunity. Their adjuvant potency is a factor of inflammasome activation and enhanced antigen cross-presentation by dendritic cells (DCs), but how antigen cross-presentation is induced is not clear. Here we show that SBAs uniquely induce intracellular lipid bodies (LBs) in the CD11b + DC subset in vitro and in vivo. Using genetic and pharmacological interference in models for vaccination and in situ tumour ablation, we demonstrate that LB induction is causally related to the saponin-dependent increase in cross-presentation and T-cell activation. These findings link adjuvant activity to LB formation, aid the application of SBAs as a cancer vaccine component, and will stimulate development of new adjuvants enhancing T-cell-mediated immunity.

\footnotetext{
${ }^{1}$ Department of Tumor Immunology, Radboud Institute for Molecular Life Sciences, Radboud UMC, Geert Grooteplein 26, 6525 GA Nijmegen, The Netherlands. ${ }^{2}$ Department of Anesthesiology, Pain and Palliative Medicine, Radboud UMC, Geert Grooteplein 10, 6525 GA Nijmegen, The Netherlands. ${ }^{3}$ Institute for Laboratory Medicine, Clinical Chemistry and Pathobiochemistry, Charité Universitätsmedizin, Augustenburger Platz 1, 13353 Berlin, Germany, ${ }^{4}$ INSERM, Institut Curie, Section Recherche, Rue d'Ulm 26, 75005 Paris, France. ${ }^{5}$ MSD Animal Health, Wim de Korverstraat 35, 5831 AN Boxmeer,

The Netherlands. Correspondence and requests for materials should be addressed to G.J.A. (email: Gosse.Adema@radboudumc.nl).
} 
A djuvants are diverse compounds that enhance the effectiveness of (cancer) vaccines. For human vaccines, registered adjuvants are limited and include aluminumbased and oil/water-based adjuvants. These adjuvants induce robust antibody responses, but weak cell-mediated immunity, which is crucial for application in anti-cancer vaccines. Saponin-based adjuvants (SBAs) are promising new adjuvants that enhance T-cell-mediated immunity.

Saponins are a large family of amphipathic plant glycosides, structurally sharing a lipophilic triterpene derivative. By purification of the raw plant material, fractions with distinct immunostimulatory properties and safety profiles have been identified $^{1}$. Although most saponins have strong binding affinity for cholesterol, only some stimulate the immune system $^{2}$. Forty nanometer cage-like particles called immune stimulatory complexes (ISCOMs) form when immunoactive saponin, cholesterol and phospholipid are brought together and dialysed $^{3}$. The physical properties of ISCOM adjuvants contribute to its stability, and reduce the haemolytic effects associated with saponins. SBAs stimulate strong innate and adaptive cellular immunity, and elicit humoral responses of all $\mathrm{IgG}$ isotypes with a mixed Th1/Th2 balance ${ }^{4-6}$. By contrast, aluminum-based or oil/water-based adjuvants predominantly drive Th2 responses. Th1 responses are particularly vital for the clearance of viral infections, but also for the eradication of cancer ${ }^{7}$.

SBAs are now being applied in human vaccines, and several clinical trials have proven safety and efficacy ${ }^{4,5,8-9}$. Next to viral antigens (for example, H5N1), SBAs have been shown to facilitate responses to cancer antigens ${ }^{10}$. We previously demonstrated in our model of in situ tumour ablation that co-injection of SBAs induced superior anti-tumour immunity relative to other adjuvants ${ }^{11}$. This unique effect was accompanied by high numbers of cytotoxic $\mathrm{T}$ cells (CTL) specific for antigens in the ablated tumour material. Vaccination with NY-ESO-1 (ref. 12) or MAGE ${ }^{13}$ tumour antigens formulated in SBAs, induced strong humoral and T-cell-mediated immune responses in melanoma patients, leading to reduced relapse rates. Cebon and co-workers combined SBA vaccination with low doses of cyclophosphamide to deplete Treg cells, resulting in significantly increased T-cell responses in these patients ${ }^{14}$. These findings, and the improving safety profile of $\mathrm{SBAs}^{15}$, place SBAs at the forefront of current anti-cancer vaccine research.

Despite the growing need to understand how vaccines work, the exact immunological mechanisms of many classical immune adjuvants are not well defined. Wilson et al. ${ }^{16}$ demonstrated the involvement of the NLRP3 inflammasome and IL-18 production in ISCOM-mediated vaccine efficacy; however, this finding does not fully explain why ISCOMs stand out from other adjuvants that trigger the inflammasome (for example, $\mathrm{Al}(\mathrm{OH})_{3}$ ). Dendritic cells (DCs) have a critical role in the in vivo effectiveness of SBA-aided vaccines, and enhanced antigen cross-presentation by DCs has been reported to be particularly important for the ability of SBAs to induce cellular CD8 + T-cell immunity ${ }^{17}$. Two main intracellular pathways for the cross-presentation of exogenous antigens have been proposed, referred to as the 'cytosolic' and 'vacuolar' pathways ${ }^{8}$. In the cytosolic cross-presentation pathway, internalized proteins are slowly degraded in endosomal compartments by enzymatic digestion at acidic $\mathrm{pH}$. By unknown mechanisms the antigens gain access to the cytosol where they are further degraded in a proteasome dependent manner. Peptides generated this way can then enter the classical MHC-I presentation route. By contrast, cross-presentation through the vacuolar pathway is proteasome independent, but sensitive to blockade of lysosomal proteolysis. Antigen processing and loading on MHC-I therefore occurs in endocytic compartments only. Cross-priming will only occur in DCs matured by interaction with pathogen-associated molecular patterns or $\mathrm{CD} 4+\mathrm{T}$ helper cells ${ }^{19}$. Next to activation status, the sub-class of DC is also of importance. DCs can be classified as conventional DCs ( $\mathrm{cDCs}$ ), plasmacytoid DCs (pDCs) or monocyte-derived DCs ${ }^{20}$. $\mathrm{cDCs}$ are located in lymphoid and non-lymphoid tissues and can be further classified into two ontogenetically distinct subtypes: the $\mathrm{CD} 8 \alpha+/ \mathrm{CD} 103+\mathrm{DCs}$ and the $\mathrm{CD} 11 \mathrm{~b}+\mathrm{DCs}^{21,22}$. In vivo studies suggest that cross-presentation is typically performed by the $\mathrm{CD} 8 \alpha+/ \mathrm{CD} 103+$ subset of $\mathrm{CDCs}^{23,24}$; however, under specific conditions every other subtype is capable of crosspresentation $^{25}$. For instance, in an inflammatory environment in vivo $\mathrm{CD} 11 \mathrm{~b}+$ monocyte-derived DCs efficiently cross-present OVA protein expressed by Escherichia coli (ref. 26). In our tumour models, SBAs trigger an unprecedented level of cross-presentation ${ }^{11}$, but how SBAs steer this process is unknown.

Lipid body (LB) organelles consist of a phospholipid monolayer that surrounds a core of neutral lipids, such as sterol esters or triacylglycerols. The monolayer contains numerous proteins, many with unknown function ${ }^{27}$. Enzymes of lipid metabolism (for example, diacylglycerol acyltransferase, DGAT), LB membrane proteins (for example, adipose differentiationrelated protein, $\mathrm{ADRP}^{28}$ ), and ER proteins (for example, the $\mathrm{p} 47$ GTPase IGTP) have been identified in LB fractions ${ }^{29,30}$. Ralph Steinman made note of LBs in his seminal 1973 manuscript describing the dendritic cell, but their function in immune cells is only just starting to be explored ${ }^{31,32}$.

Our data now uncover an essential link between LBs and cross-presentation in the working mechanism of SBA adjuvants occurring uniquely in the CD11b + DC subset. Genetic or pharmacological blockade of LB induction effectively abrogates the SBA-induced antigen cross-presentation, in a vaccination setting as well as an in situ tumour ablation model. Mechanistic studies suggest that SBAs induce LBs independent of SBA-triggered antigen translocation, and that LBs facilitate the proteasomal route of cross-presentation. These data highlight the importance of lipid body induction in CD11b + DC for SBA vaccine activity, will aid SBA vaccine development, and the design of new adjuvants enhancing T-cell-mediated immunity.

\section{Results}

Saponin-based adjuvants induce cross-presentation in DCs. SBAs induce potent immune responses to a broad repertoire of antigens. SBAs and antigen can just be ad-mixed without prior complexation or formulation ${ }^{4,5}$, or simply be injected in an ablated tumour to boost antigen-specific CD8 + T-cell responses $(\text { Fig. } 1 \mathrm{a}, \mathrm{b})^{11}$. In an ablation setting, the tumour debris that stays inside the body following treatment provides an effective source of (neo)antigens for processing by the immune system ${ }^{33,34}$. The induction of potent CD8 + T-cell responses is dependent on the ability of SBAs to facilitate cross-presentation of exogenous antigens in MHC-I by DCs ${ }^{5,35,36}$. How SBAs act to induce antigen cross-presentation is poorly understood. By testing a panel of classical non-microbial vaccine adjuvants, we first confirmed that the saponin containing ISCOM Matrix $C$ induced a dramatic increase in ovalbumin (OVA) antigen cross-presentation in GM-CSF-propagated DCs (Fig. 1c; Supplementary Fig. 1a). As a read out system, we used either the co-stimulation independent B3Z reporter T-cell line ${ }^{29}$, or OVA-specific T cells from OT-I transgenic mice. None of the other non-microbial vaccine adjuvants was able to induce cross-presentation of OVA in DCs (Fig. 1d). The low amount of OVA used did not by itself lead to detectable cross-presentation in DCs in our assays (Fig. 1d), and 
a

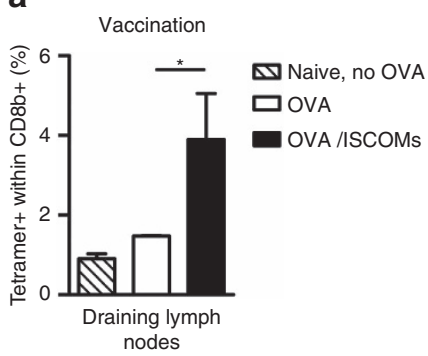

b

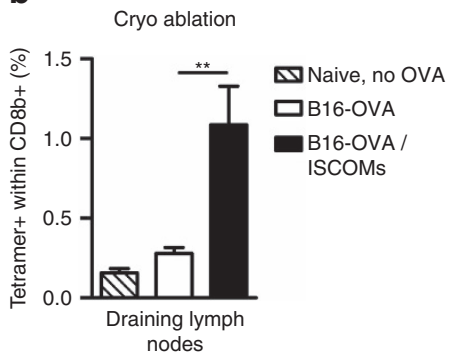

c

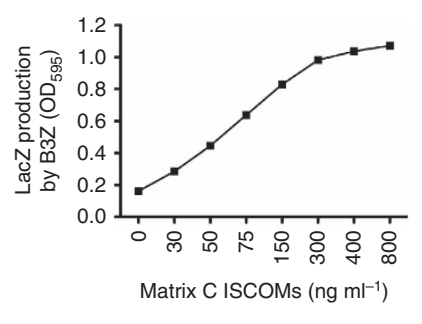

d

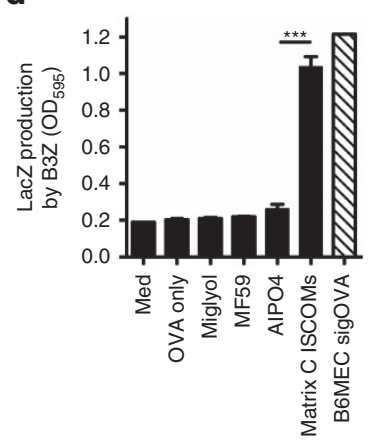

e

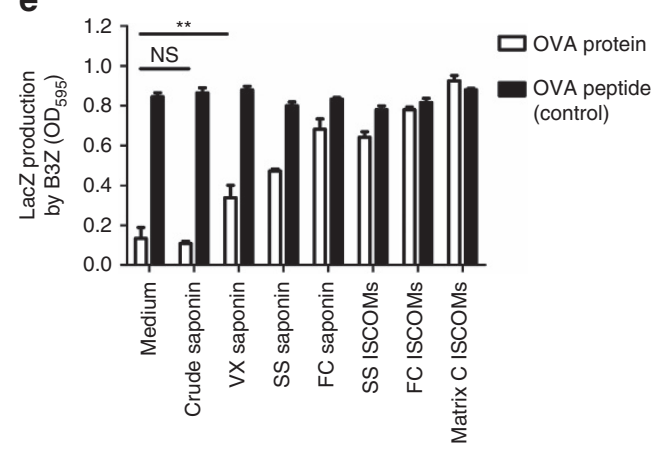

Figure 1 | Saponin-based adjuvants induce cross-presentation in dendritic cells. (a,b) Tetramer staining of OVA specific T cells in draining lymph nodes, 10 days following s.c. injection of $20 \mu \mathrm{g}$ OVA and/or $30 \mu \mathrm{g}$ ISCOMs (a, vaccination), or 7 days following co-treatment of established B16OVA melanomas (5-8 mm diameter) with in situ tumour ablation and $30 \mu$ g peritumourally injected ISCOMs (b, Cryo-ablation). (c,d) In vitro B3Z cross-presentation assays demonstrating a concentration-dependent increase in cross-presentation of OVA, only when Matrix C ISCOMs was added. GM-CSF-cultured BMDCs were exposed to the indicated compounds and $80 \mu \mathrm{g} \mathrm{ml} \mathrm{m}^{-1}$ OVA protein for $5 \mathrm{~h}$, washed and co-cultured o/ $\mathrm{n}$ with B3Z cells, which produce $\beta$-galactosidase upon recognition of the OVA peptide in $\mathrm{K}^{\mathrm{b}}$. Data in (d) are excerpts from larger titrations. For the non-reactive adjuvants the maximum concentration used is shown $\left(1.5 \mathrm{H} \mathrm{ml}^{-1}\right)$, for the ISCOMs the concentration shown is $400 \mathrm{ng} \mathrm{ml}^{-1}$. (e) In vitro cross-presentation after $5 \mathrm{~h}$ exposure to $400 \mathrm{ng} \mathrm{ml}-1$ of various $\mathrm{SBAs}$ or controls. This contains a crude saponin mixture not purified for immunoactive fractions, commercially available immunoactive saponin fractions (VX, SS and FC) and ISCOM structures made with these saponins according to an 'open access' protocol. Passive peptide loading with OVA $\mathrm{K}^{\mathrm{b}}$ peptides was used to demonstrate viability and/or equal MHC-I levels. Similar results were obtained in two to three independent experiments. Statistical analysis was done using a one or two-way ANOVA with post hoc Bonferroni or Tukey tests.

MHC-II-restricted antigen presentation of OVA to OT-II cells was not altered by SBA administration (Supplementary Fig. 1b).

Only very distinct saponin fractions show immunological activity and qualify as vaccine adjuvant. So far, most SBA cross-presentation studies have been performed using proprietary made ISCOM structures ${ }^{17,35,37-39}$. Therefore, we tested whether different immune activating saponin fractions exhibited the same capacity to induce cross-presentation. All three commercially available fractions tested, and the corresponding ISCOMs made from two of these fractions according to an open-access protocol (Supplementary Fig. 2 and ref. 40) led to efficient increases in cross-presentation (Fig. 1e). In contrast, a saponin mixture not enriched for immuno-active fractions (crude saponin) did not induce cross-presentation, also at higher concentrations (Fig. 1e). Importantly, SBA treatment did not lead to reduced cell viability or MHC-I levels on the surface of the APCs, as presentation of pulsed peptide was intact in all occasions (Fig. 1e; Supplementary Fig. 3). Finally, we confirmed that within the ISCOMs the saponins are the cross-presentation inducing moiety, and not the ISCOM-stabilizing component cholesterol (Supplementary Fig. 4).

Cross-presentation induction in DCs thus is a feature shared by immunoactive saponins, but not by other saponins.

SBA-induced cross-presentation is co-stimulation independent. The increases in cross-presentation could not be explained by differential surface expression of the mannose receptor (CD206, involved in uptake of OVA), or MHC-I/II (Fig. 2a), and consistent with literature, SBAs also did not change CD80 or CD86 levels on the in vitro cultured DCs (Fig. 2b) ${ }^{17}$. Together with the fact that $\mathrm{B} 3 \mathrm{Z}$ cells react co-stimulation independent, this indicated that maturation status of the DCs is not causing the observed increases in cross-presentation. In line with this, BMDCs from Tlr4-/-, Myd88-/ - or Trif-/ - mice all had an intact ability to crosspresent OVA following ISCOMs treatment (Fig. 2c). Moreover, also the type I IFN receptor or the inflammasome component NLRP3 did not affect SBA-aided cross-presentation (Fig. 2c). Antigen uptake or overall antigen processing by GM-CSF DCs, as measured by the internalization of fluorescent OVA or breakdown of DQ-OVA, was not altered following SBA treatment (Fig. 2d).

We conclude that the increases in cross-presentation induced by SBAs in DCs are antigen uptake or co-stimulation independent.

Endosomal ROS is not involved in cross-presentation by SBAs. As previous work has implicated a role for reactive oxygen species (ROS) produced by the phagosomal NADPH-oxidase (NOX) complex in cross-presentation ${ }^{41}$, we set out to determine cellular ROS levels following SBA exposure. Employing two probes for detection of total ROS levels in our DCs (DHR123 and $\mathrm{H}_{2}$ DCFDA), we found that SBA treatment for $5 \mathrm{~h}$ (similar to the exposure time in the cross-presentation assays) did not increase ROS levels, while the control PMA did (Fig. 2e). Although ceasing ROS production by co-treatment of the cells with commonly used 
a
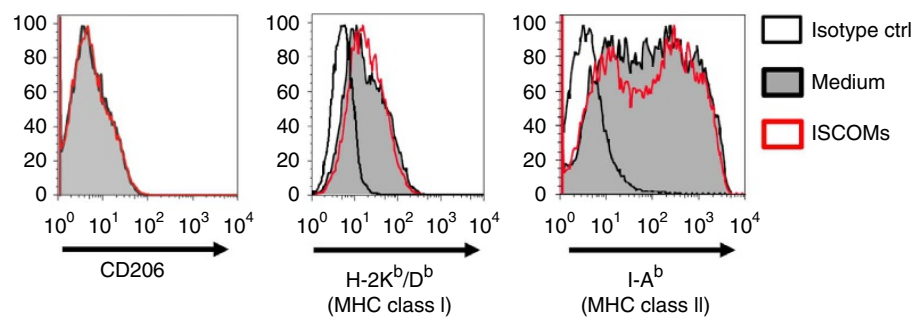

b

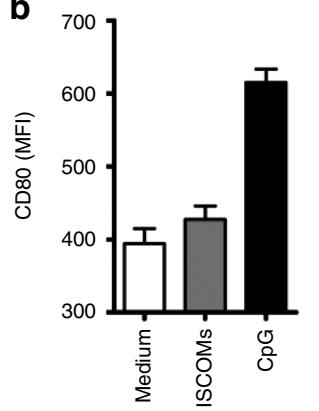

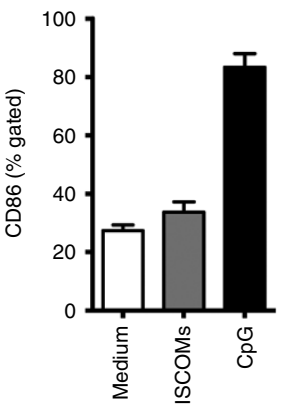

NLRP3

c
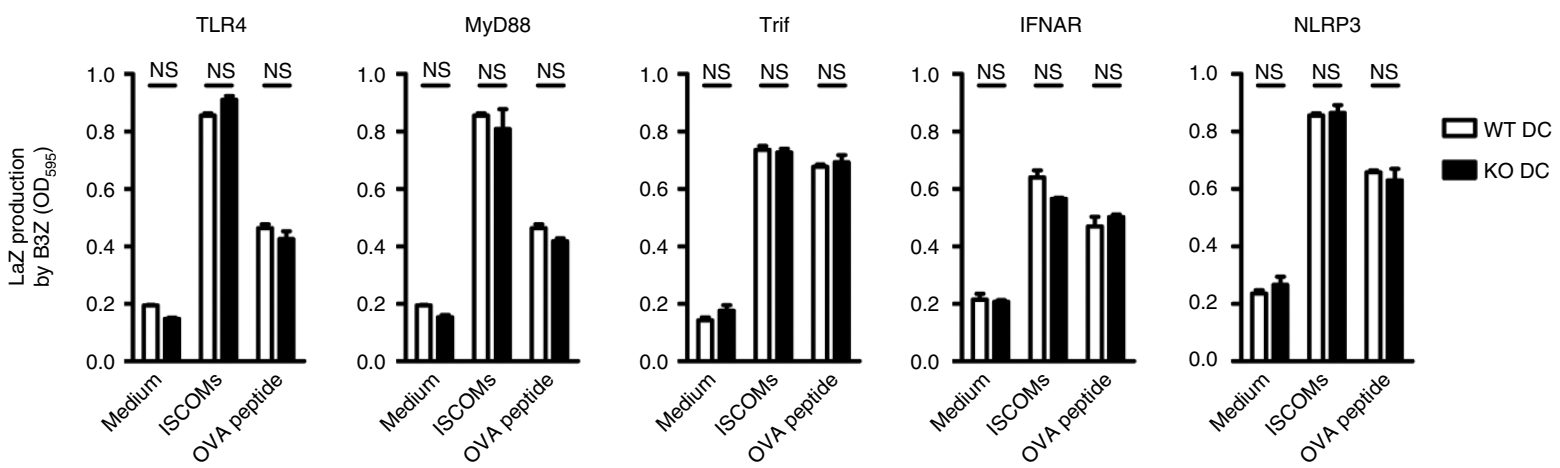

d

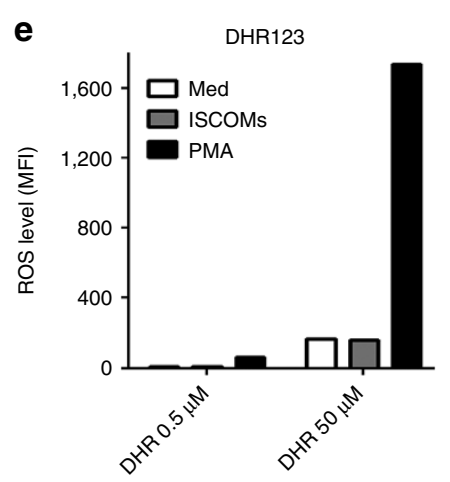

g GP91phox
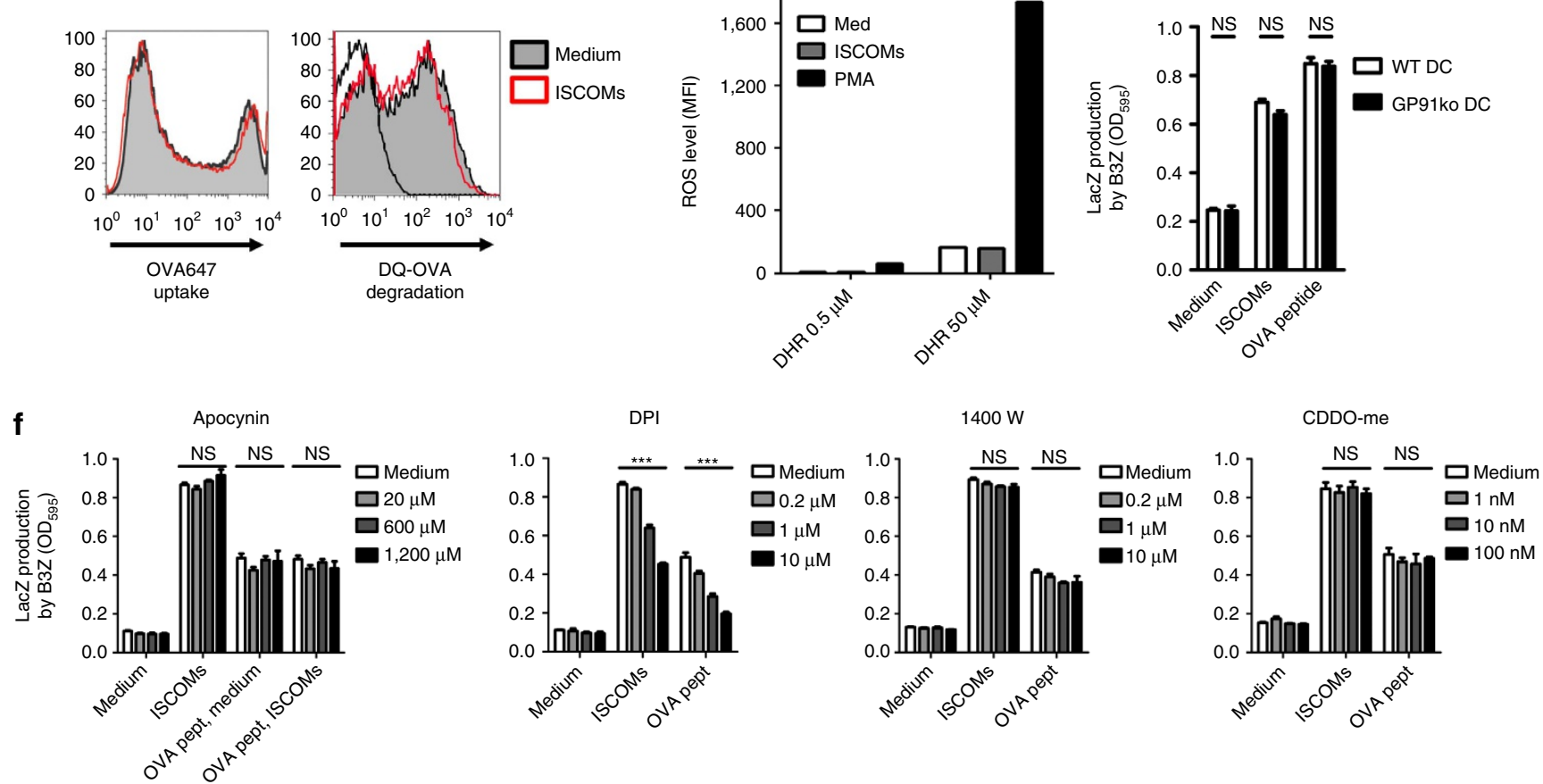

Figure 2 | SBA-induced cross-presentation is independent of co-stimulation or ROS. (a) FACS analysis of surface markers CD206 (mannose receptor), MHC-I, and MHC-II. GM-CSF BMDCs were exposed for $5 \mathrm{~h}$ to ISCOMs or medium, and processed for FACS staining. Open black lines represent the corresponding isotype controls. Filled grey lines show the medium-treated cells, while the open red lines are the ISCOM treated samples. (b) FACS analysis of DC maturation markers CD80 and CD86 after $18 \mathrm{~h}$ stimulation with ISCOMs or $1 \mu \mathrm{g} \mathrm{ml}{ }^{-1} \mathrm{CpG}$. (c) Bone-marrow of indicated knockout mice was used to generate GM-CSF BMDCs. Cells were exposed for $5 \mathrm{~h}$ to medium or ISCOMs, washed and co-cultured o/ $\mathrm{n}$ with B3Z cells. External peptide pulsing $\left(5 \mathrm{p} \mathrm{ml}^{-1}, 30 \mathrm{~min}\right.$ ) was used to control for viability and/or MHC-I levels. (d) Antigen uptake and processing as analysed by FACS. BMDCs were given $0.25 \mathrm{mg} \mathrm{ml}^{-1}$ OVA coupled to the fluorophore Alexa647, or $1 \mu \mathrm{g} \mathrm{ml} \mathrm{l}^{-1} \mathrm{DQ}-\mathrm{OVA}$, during the $5 \mathrm{~h}$ exposure time to medium or ISCOMs. DQ-OVA will start to fluoresce once degraded. (e) Total cellular reactive oxygen species (ROS) were measured using the DHR123 probe. GM-CSF cultured BMDCs were plated in serum-free medium and pretreated for $10 \mathrm{~min}$ with 0.5 or $50 \mu \mathrm{M}$ dihydrorhodamine 123 . Next, ISCOMs or $1 \mu \mathrm{gl} \mathrm{ml}^{-1} \mathrm{PMA}$ was added and cells were incubated for $45 \mathrm{~min}$ before analysis by FACS. (f) In vitro cross-presentation in the presence of NADPH oxidase inhibitors. Indicated compounds and concentrations were added during the $5 \mathrm{~h}$ exposure period to ISCOMs. (g) In vitro cross-presentation using Gp91phox - / - bonemarrow DCs. Data in (e) represent single values in titration, whereas the other data show means from triplicates with s.e.m. Data are representative figures from two to three individual experiments. Statistical analyses were performed using two way ANOVA with post hoc Bonferroni tests. 
concentrations of the NADPH oxidase inhibitor DPI initially seemed to abrogate the SBA-induced cross-presentation, the peptide control revealed lowered MHC-I expression due to decreased cell viability or down regulation (Fig. 2f). Three alternative NADPH oxidase inhibitors and various ROS scavengers did however not interfere with SBA-induced cross-presentation (Fig. 2f; Supplementary Fig. 5). Finally, SBAs could normally induce cross-presentation in DCs deficient in the ROS-inducing NOX2 complex component Cybb (GP91phox) (Fig. 2g).

Altogether these data do not support a significant role for ROS in the induction of cross-presentation by SBAs.

SBAs induce lipid bodies in parallel with cross-presentation. Bougneres et al..$^{31}$ previously demonstrated a relationship between cross-presentation in DCs and the lipid body-residing molecule IGTP. This prompted us to examine the presence of LBs in DCs exposed to various non-microbial adjuvants. To this end, we stained cells with the neutral lipid dye Bodipy ${ }^{493 / 503}$. Strikingly, SBAs (FC saponin, ISCOMs) appeared to increase the number and the brightness of cytosolic LBs as determined by confocal microscopy (CLSM), always in perfect correlation with crosspresentation levels (Figs 1d and 3a,b). Co-staining for the LB protein ADRP confirmed that the detected lipid aggregates were LBs (Fig. 3c). Other adjuvants like AlPO4, non-immunogenic crude saponin or OVA alone could not elevate the number of LBs (Fig. 3b). A time course demonstrated that the numbers of LBs per cell increased linearly over the first $32 \mathrm{~h}$, which again strongly correlated with cross-presentation levels (Fig. 3d-f). Control experiments confirmed that the saponins, but not the ISCOM particle stabilizing component cholesterol represent the active moiety inducing LBs, and that mice deficient for Tlr4, Nlrp3 or Ifnar had a normal ability to generate LBs following SBAs (Supplementary Fig. 6).

Together, these results suggest that GM-CSF DCs in vitro possess the striking ability to induce LBs following treatment with SBAs, which is tightly correlated with the ability to cross-present soluble antigen.

LB formation and cross-presentation in DCs in vivo. To determine whether in vivo vaccination with SBAs also resulted in LB formation in cross-presenting DCs, we co-injected mice with ISCOMs and OVA. Twelve hours post-injection draining lymph nodes (LNs) were isolated and subjected to $\mathrm{CD} 11 \mathrm{c}+$ magnetic bead sorting to isolate all DCs (Fig. 3g). Similarly, DCs were isolated from B16OVA tumour bearing mice after cryo-ablation treatment with SBA co-injection (Fig. 3h). Assaying the isolated CD11c + DC using two cross-presentation read-outs demonstrated that ISCOMs efficiently induced cross-presentation in DCs, but not the $\mathrm{CD} 11 \mathrm{c}$ negative fraction. Evaluation of the LB content by CSLM (Fig. 3i, left) and FACS (Fig. 3i, right) showed that also after in vivo SBA vaccination the number of LBs increased.

SBA sensitivity is a unique feature of monocytic CD11b + DCs. DCs can be differentiated in vitro from BM precursors using GM-CSF or Flt3-L. Remarkably, we found that only DCs generated with the growth factor GM-CSF, and not DCs cultured with Flt3-L, were able to cross-present antigen after SBA exposure (Fig. 4a). Increasing exposure time or dose could not change the observed cell specificity (Supplementary Fig. 7). Importantly, Flt3L DCs readily activated B3Z cells after external pulsing with OVA $\mathrm{K}^{\mathrm{b}}$ peptide (Supplementary Fig. 7b). Moreover, in these cells CD206 and MHC-I expression, and ovalbumin uptake were not influenced by the SBAs (Supplementary Fig. 7c). In line with the inability to cross-present antigen upon SBA exposure, the
Flt3-L-generated DCs were incapable of inducing LBs (Fig. 4b), even at longer exposure times.

The BM cultures with Flt3-L or GM-CSF both give rise to a complex set of DC subpopulations each arising from predetermined precursors present in the starting population ${ }^{42-44}$. The observation that only $45 \%$ of the in vitro generated GM-CSF DCs contained LBs prompted us to purify the different DC subsets using flowcytometry, and to test their response to SBAs. First, Flt3-L DCs were sorted based on Naik et al. ${ }^{43}$ (Fig. 4c). In the three resulting DC subpopulations no population capable of cross-presenting OVA upon SBA exposure could be detected (Fig. 4d). GM-CSF DCs were sorted based on Helft et al. $^{42}$, with modifications (Fig. 4e). Strikingly, only the CD135 (Flt3) negative DCs (MHCII ${ }^{\text {lo }} \mathrm{CD} 11 b^{\text {hi }} \mathrm{CD} 115^{\text {hi }} \mathrm{CD} 135^{\text {neg }}$ and $\mathrm{MHCII}{ }^{\text {int }} \mathrm{CD} 11 \mathrm{~b}^{\text {int }} \mathrm{CD} 115^{\text {int }} \mathrm{CD} 135^{\text {neg }}$ subpopulations) could efficiently cross-present OVA in response to SBAs, whereas the $\mathrm{CD} 135$ positive DCs $\left(\mathrm{MHCII}{ }^{\mathrm{hi}} \mathrm{CD} 11 \mathrm{~b}^{\mathrm{int}}\right.$ $\mathrm{CD} 115^{\text {low }} \mathrm{CD} 135^{\text {pos }}$ subset) could not (Fig. 4f). Since the $\mathrm{MHCI}^{\text {int }} \mathrm{CD} 11 \mathrm{~b}^{\text {int }} \mathrm{CD} 115^{\text {int }} \mathrm{CD} 135^{\text {neg }}$ cells are considered multipotent intermediates ${ }^{42}$, we focused on the two differentiated DC populations. Again, SBA-mediated induction of lipid bodies only occurred in the DC subset cross-presenting antigens due to SBAs: at least $80 \%$ of the $\mathrm{MHCII}^{\mathrm{lo}} \mathrm{CD} 11 \mathrm{~b}^{\text {hi }}$ CD115 hi CD135 neg DCs now contained LBs after $5 \mathrm{~h}$ (Fig. $4 \mathrm{~g}$ ). These SBA responsive DCs were previously shown to be derived from monocytic precursors ${ }^{42}$. As apparently the monocytic precursors in the Flt3-L culture did not yield SBA responsive DCs, the Flt3-L culture was supplemented with GM-CSF for the last two days. Strikingly, the Flt3-L DCs now acquired full ability to cross-present antigens in response to SBAs (Fig. 4h). Sorting experiments confirmed that CD11b $+(\operatorname{Sirp} \alpha+) \mathrm{DCs}$, but not the $\mathrm{CD} 8 \alpha+(\mathrm{CD} 24+)$ DCs, were responsible for SBA-induced cross-presentation (Fig. 4i; Supplementary Fig. 8). Similarly, analysis of $\mathrm{pDCs}$, and $\mathrm{CD} 8 \alpha+$ or $\mathrm{CD} 11 \mathrm{~b}+\mathrm{cDCs}$ from Flt3-L cultures that were first sorted and then individually exposed to GM-CSF confirmed that only the CD11b + DCs gained the capacity to respond to SBAs (Fig. 4i). Stainings for the monocytic marker CD115 showed increased presence of CD115+ cells among the SBA-responsive CD11b + DCs following GM-CSF exposure (Supplementary Fig. 8).

Collectively, these results define GM-CSF-sensitized CD11b + DCs, presumably from monocytic origin, to be the only cells responsive to SBAs.

Only monocytic CD11b + DCs respond to SBAs in vivo. Next, we determined whether the selective SBA responsiveness of in vitro cultured DCs also exists in vivo. $\mathrm{CD} 8 \alpha+$ and $\mathrm{CD} 11 \mathrm{~b}+$ DCs sorted from naive spleens based on the markers CD24, Sirp- $\alpha$, CD115 and CD4 (Fig. 5a) ${ }^{36}$, could not cross-present OVA upon SBA exposure but efficiently presented externally loaded control peptides (Fig. 5b). This is not unexpected as no CD115 staining was observed on these $\mathrm{CD} 11 \mathrm{~b}+\mathrm{DCs}$, indicating the absence of cells from monocytic origin, shown to be responsive to SBA in vitro (Fig. 5a). Therefore, resident and migratory $\mathrm{CD} 8 \alpha+, \mathrm{CD} 103+$ or $\mathrm{CD} 11 \mathrm{~b}+\mathrm{DCs}$ were sorted from naïve LNs (Fig. $5 c)^{21}$. Subsequent exposure to SBAs demonstrated that $\mathrm{LN}$ resident, but especially the $\mathrm{LN}$ migratory $\mathrm{CD} 11 \mathrm{~b}+\mathrm{DCs}$, were capable of cross-presenting OVA (Fig. 5d). The ability to respond to SBAs coincided with the presence of cells expressing the monocytic markers CD14 and Ly6C within the resident, but especially the migratory CD11b + DCs (Supplementary Fig. 9). To evaluate a situation with a high monocytic influx, we analysed mice bearing Flt3-L excreting tumours to provoke expansion of endogenous DCs, including pDCs, in an activated environment ${ }^{45,46}$. From the tumour-draining LNs we isolated the pDC 
a

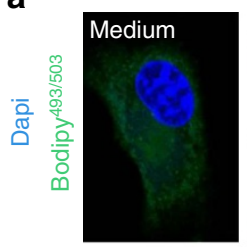

c

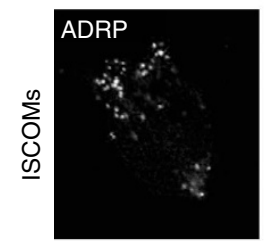

d

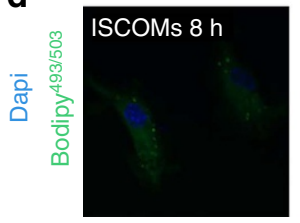

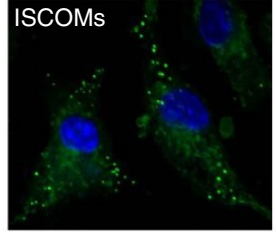

\section{Bodipy}
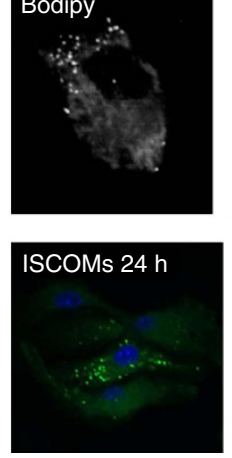

b
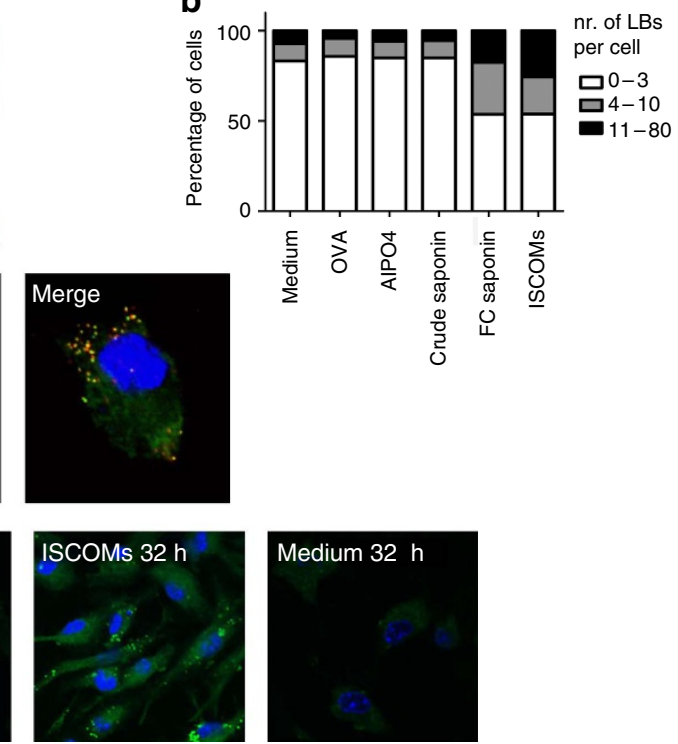

Medium $32 \mathrm{~h}$

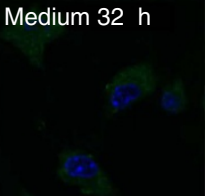

e

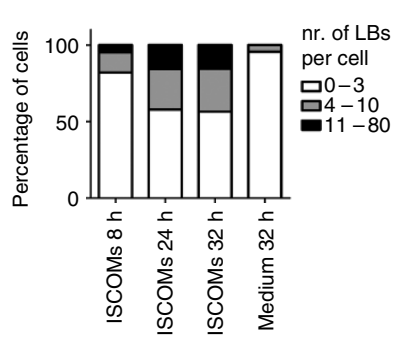

h

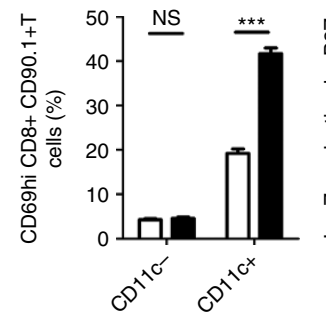

f

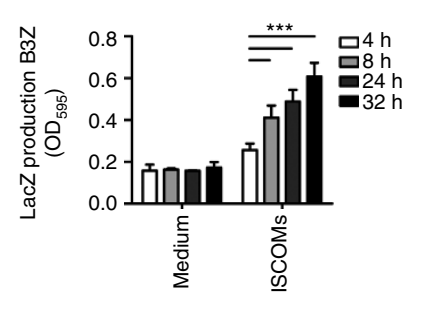

g
Lymph nodes

in vivo exposure to SBAs
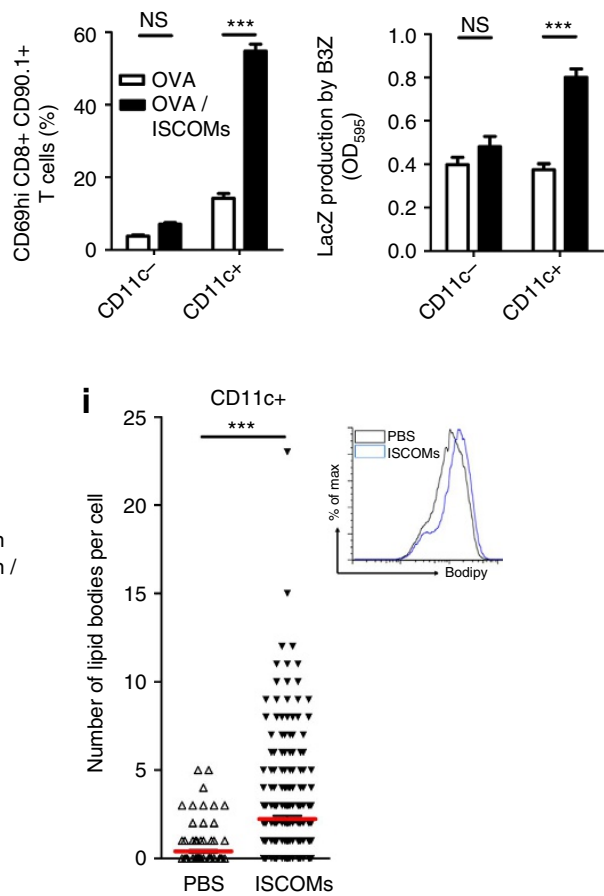

Figure 3 | SBAs induce in vitro and in vivo lipid bodies in correlation with cross-presentation. (a-c) GM-CSF-cultured BMDCs following $5 \mathrm{~h}$ exposure to the indicated adjuvants were fixed and stained with the LB probe Bodipy ${ }^{493 / 503}$. Nuclei were counterstained with DAPI, and cells were analysed by confocal microscopy. (a) CLSM image showing SBA-induced LBs. (b) Quantification of lipid bodies following exposure to OVA alone, AIPO4, non-immunoactive saponin, FC saponin, or ISCOMs. Numbers of lipid bodies per cell were obtained from 90-150 cells found in 15 randomly taken CLSM images, and divided over three strata. (c) LB staining with counterstaining for ADRP (d-f) $32 \mathrm{~h}$ time-course showing concomitant increases in LB numbers (d,e) and cross-presentation (f). $(\mathbf{g}, \mathbf{h})$ In vitro cross-presentation assay on isolated lymph node CD11c + DCs using OT-I cells (left) or B3Z cells (right) as a readout. (g) Three hundred microgram endotoxin-free OVA protein was injected s.c. on the femur of WT C57BI/6 mice, with or without $30 \mu \mathrm{g}$ ISCOMs. Twelve hours later, draining lymph nodes were harvested from which CD11c + cells were isolated using MACS beads. Next, these cells were co-cultured with the reporter cells. CD69 levels on CD8 + CD90.1 + OT-I cells were measured after 1 day, while B3Z cells were analysed after 2 days. (h) Established B16OVA melanomas (5-8 mm diameter) were injected i.t. with a booster shot of OVA $(20 \mu \mathrm{g})$, after which they were treated with in situ tumour ablation and $30 \mu \mathrm{g}$ peritumourally injected ISCOMs. $12 \mathrm{~h}$ later draining lymph nodes were harvested from which CD11c + cells were isolated using MACS beads. Next, these cells were co-cultured with the reporter cells like in $\mathbf{g}$. (i) Quantification of LBs per cell using CLSM (left) or FACS (right), on ex vivo CD11c + cells as treated and isolated under ( $\mathbf{g}$ ). All data were reproduced in two to three independent experiments. Statistical analyses were performed using two-way ANOVA with post hoc Bonferroni tests, or Student's t-test for $\mathbf{i}$.

fraction, a fraction containing all CD8 $\alpha+$ LN-resident DCs, and the CD11b ${ }^{\text {hi }}$ DC fraction, and exposed them in vitro to SBAs (Fig. 5e). Clearly, only the CD11b ${ }^{\text {hi }}$ DC fraction was able to cross- present OVA upon SBA exposure (Fig. 5f). As expected, these DCs were also the only cells that contained LBs after SBA treatment (Fig. 5g). Additional FACS analysis of MHC-II levels 

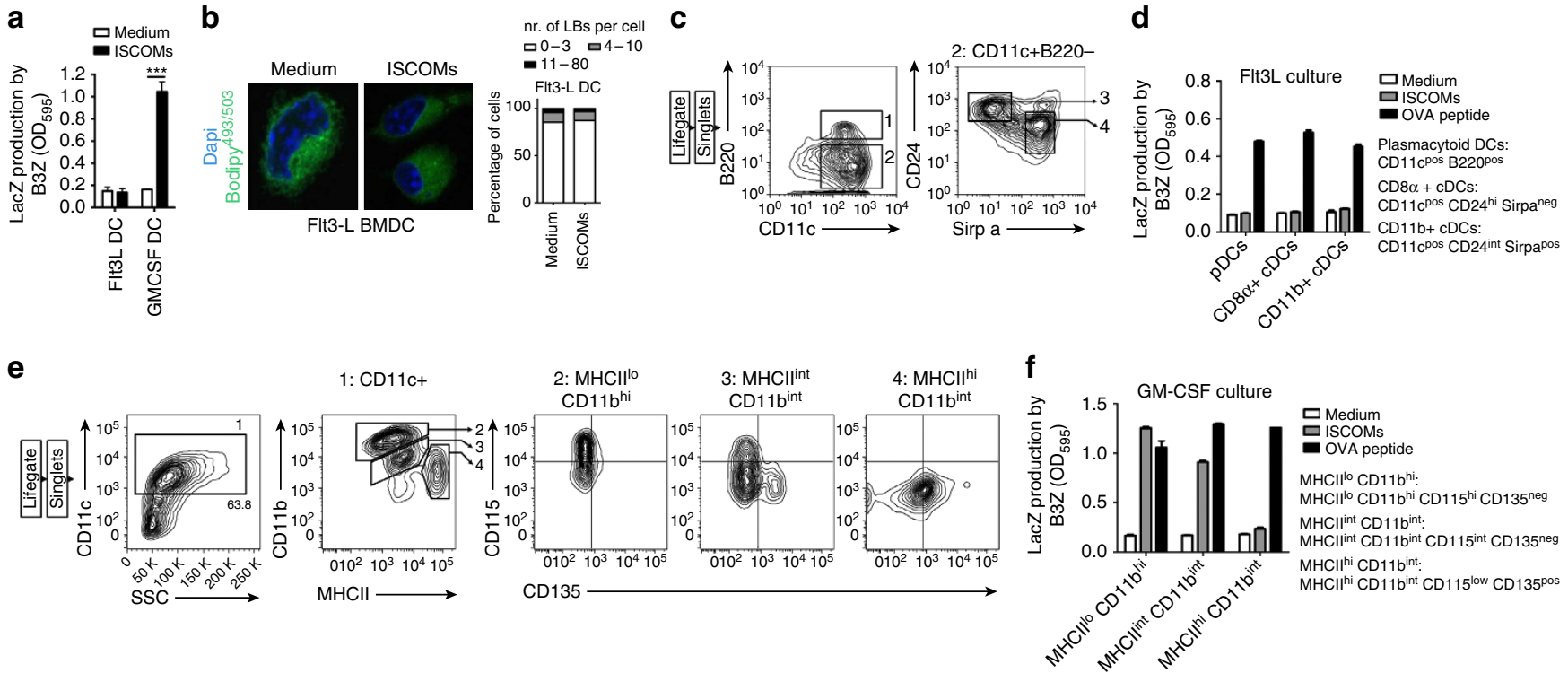

- OVA peptide

MHCIIIo CD11 bi. $^{\text {hi. }}$ MHCIllo CD11 ${ }^{\text {hi }}$ CD115 hi CD135 neg MHCIIIt CD11 $b^{\text {int: }}$ MHCII ${ }^{\text {int }} \mathrm{CD} 11 \mathrm{~b}^{\text {int }} \mathrm{CD} 115^{\text {int }} \mathrm{CD} 135^{\text {neg }}$ MHCIII $\mathrm{CD} 11 \mathrm{~b}^{\text {int }}$ MHCIII $C D 11 b^{\text {int }} C D 115^{\text {low }} C D 135^{\text {pos }}$
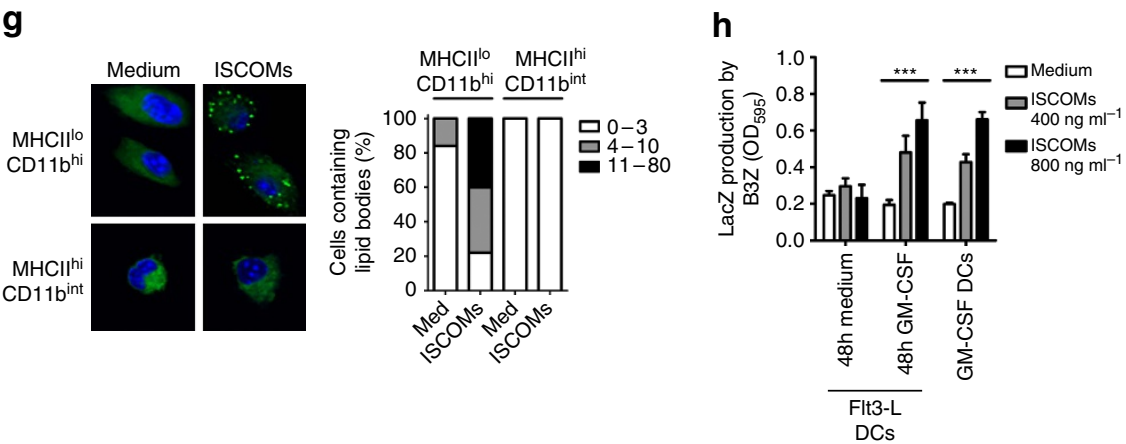

i

ㅁ Medium ㅁ ISCOMs $\square$ OVA pept
72 h GM-CSF > sort sort > 72 h GM-CSF/FIt3-L

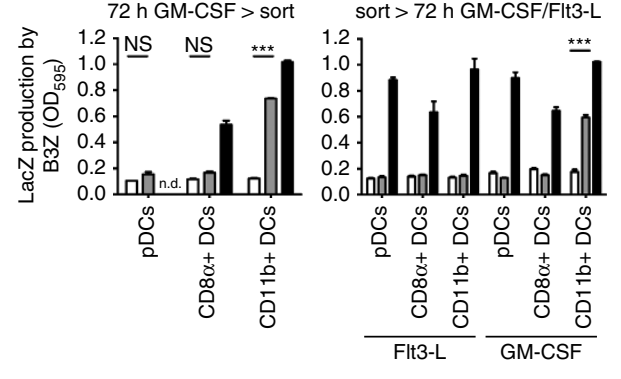

Figure 4 | Responsiveness to SBAs in vitro is unique to CD11b + DCs with monocytic origin. (a) In vitro cross-presentation assay using GM-CSFor Flt3-L-propagated DCs. (b) Confocal images with LB quantifications from Flt3-L-generated BMDCs exposed for $5 \mathrm{~h}$ to medium or ISCOMs. (c) Gating strategy of Flt3-L DCs. Sorted populations are gate 1 (pDCs), gate $3(C D 8 \alpha+D C s)$ and gate 4 (CD11b + DCs). (d) In vitro cross-presentation assay using the populations as sorted under c. (e) Gating strategy of GM-CSF DCs. Sorted populations are gate 2 (MHCII ${ }^{\text {lo }}$ CD11b ${ }^{\text {hi }}$ ), gate 3 (MHCII ${ }^{\text {int }}$ CD11b ${ }^{\text {int }}$ ) and gate $4\left(\mathrm{MHCI}^{\text {hi }} \mathrm{CD}_{11} \mathrm{~b}^{\text {int }}\right)$. (f) In vitro cross-presentation assay using the populations as sorted under e. (g) CLSM image and LB quantification of populations as sorted under $\mathbf{e}$, after $5 \mathrm{~h}$ exposure to ISCOMs or medium. (h) In vitro cross-presentation assay on Flt3-L- or GM-CSF-cultured bonemarrow DCs. In the 10 day Flt $3-\mathrm{L}$ cultures, medium was supplemented for the last $48 \mathrm{~h}$ with $20 \mathrm{ng} \mathrm{ml}^{-1} \mathrm{GM}$-CSF or medium. (i) In vitro cross-presentation assays on Flt3-L-cultured DCs, receiving GM-CSF for $72 \mathrm{~h}$ after the end of culture. In the left panel the entire day 10 cultures were supplemented with GM-CSF for another $72 \mathrm{~h}$, after which the resulting populations were sorted (Supplementary Fig. 8). In the right panel the day 10 cultures were sorted, after which the individual populations were treated with fresh Flt3-L or GM-CSF. All data were reproduced in two to three independent experiments. Statistical analyses were performed using two-way ANOVA with post hoc Bonferroni tests.

showed that these cells were highly activated, making it difficult to discriminate migratory from resident $\mathrm{CD} 11 \mathrm{~b}+$ cells within this gate (Fig. 5e). As an additional control, we showed that the $\mathrm{CD} 8 \alpha+$ DC containing fraction was able to cross-present OVA incorporated in nanoparticles, as shown previously (Fig. 5f). Finally, we sorted cells from mice treated by in situ tumour ablation with co-injection of the TLR9 ligand CpG-ODN. This procedure induces the influx of fresh (inflammatory) monocytes into the LNs, but not pDCs like with the Flt3-L excreting tumours. Similar to our former findings, only the CD11b ${ }^{\text {hi }}$ DCs could react to SBAs (Supplementary Fig. 10).

Thus, also in vivo monocytic CD11b + cells uniquely harbor the ability to cross-present soluble antigens and to induce LBs upon exposure to SBAs.

SBA-induced lipid bodies are critical for cross-presentation. To investigate whether a causal relationship exists between LBs and cross-presentation, we searched for ways to cease LB formation.
Blocking formation of triacylglycerol, fueling the LBs, by inhibiting the rate-limiting enzymes acyl-CoA, diacylglycerol acyltransferase (DGAT) 1 and 2 or by inhibiting acyl-CoA synthetase (ACS) or acetyl-CoA carboxylase (ACC), which are further upstream in lipid

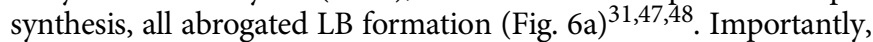
inhibiting LB formation also ended the SBA dependent capacity of DCs to cross-present antigen (Fig. 6b). Viability of the cells and MHC-I levels were not affected as confirmed by external peptide loading (Fig. 6b). To further strengthen these findings, and to exclude off-target effects of pharmacological inhibition we made use of bone-marrow DCs derived from IGTP knockout mice, having a reduced capacity to generate functional $\mathrm{LBs}^{31}$. To screen for LB induction in a higher number of cells, we established a FACS-based protocol (Fig. 6c). As expected, Igt $t p-/-$ DCs had significantly less LBs compared with wild-type mice (Fig. 6d). Although Bougneres et al. showed that IFN $\gamma$ increases expression of IGTP, and likely amplifies the molecular effects of a deficiency in IGTP $^{31}$, the SBA-induced difference was not significantly more apparent when cells were co-treated with IFN $\gamma$. Consistent with 
a

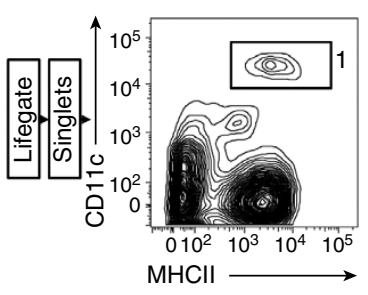

1: $\mathrm{CD} 11 \mathrm{C}^{\mathrm{hi}} \mathrm{MHC} \mathrm{C}^{\mathrm{hi}}$

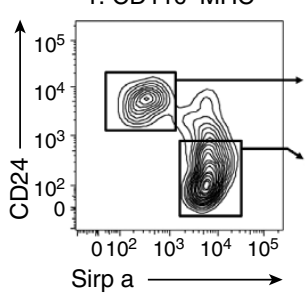

C

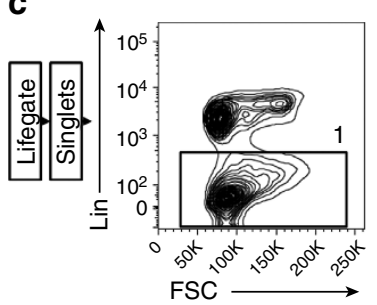

2: Resident
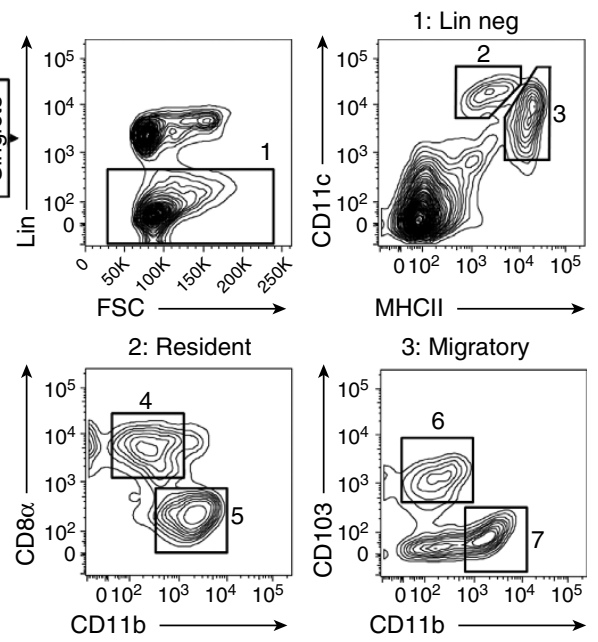

3: $\operatorname{Sirpa}^{\text {pos }} \mathrm{CD} 24^{\text {neg }}$

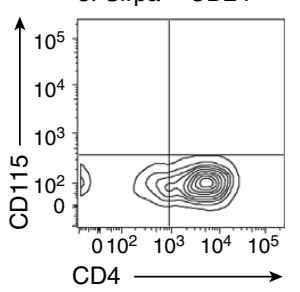

b

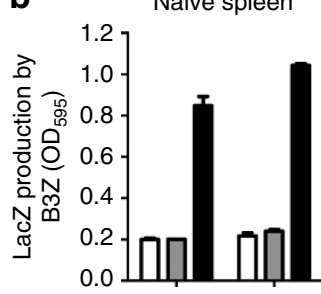

$\square$ Medium

ㅁISCOMs

- OVA peptide

CD $8 \alpha+$ DCs:

Sirponeg CD24pos

CD11b+ DCs:

Sirpo ${ }^{\text {pos }} C D 24^{\text {neg }} C D 115^{\text {neg }} C D 4^{\text {pos }}$
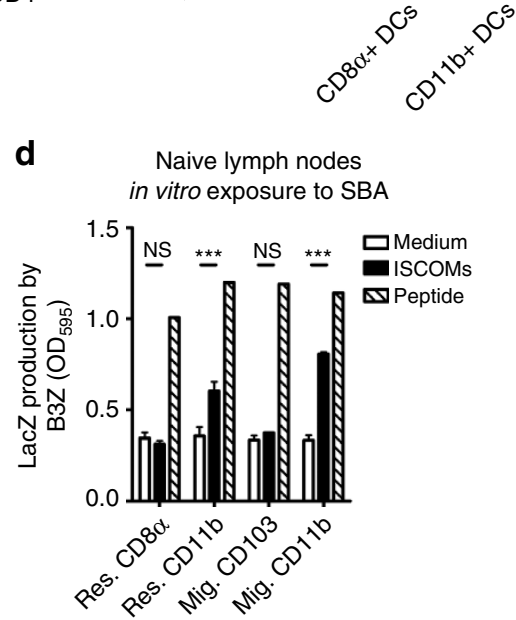

e

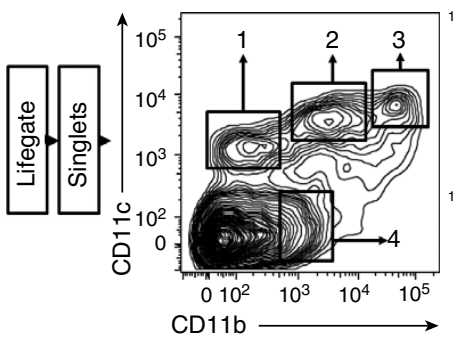

1: $\mathrm{CD} 11 \mathrm{c}^{\mathrm{int}} \mathrm{CD} 11 \mathrm{~b}^{\text {lo }}$ 2: CD11 ${ }^{\text {hi }} \mathrm{CD} 11 \mathrm{~b}^{\text {int }}$ 3: $C D 11 c^{\text {hi }} C D 11 b^{\text {hi }}$

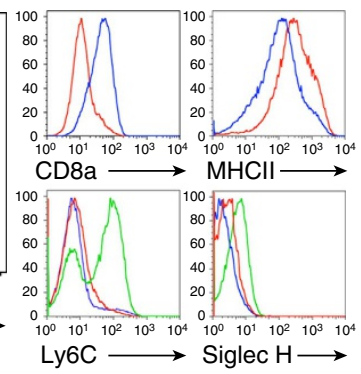

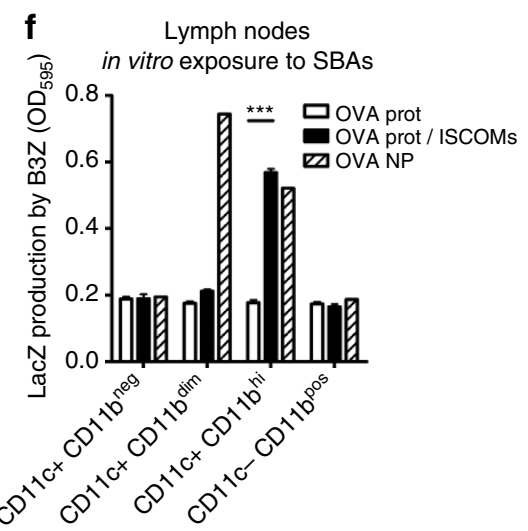

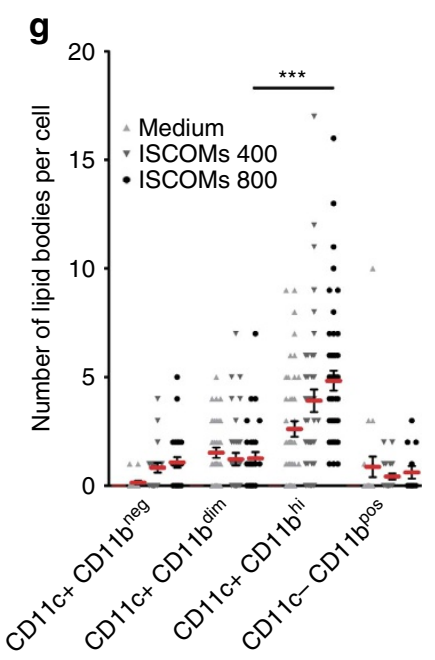

Figure 5 | Lymph node CD11b + DCs uniquely possess the ability to respond to SBAs in vivo. (a) Gating strategy for naive splenocytes. Sorted populations are gate $2(C D 8 \alpha+D C s)$ and gate $3(C D 11 b+D C s)$. (b) In vitro cross-presentation assay using the populations as sorted under a. (c) Gating strategy for naïve lymph nodes. Lin- was defined as B220- and Ly6G-. Sorted populations are gate 4 (LN resident CD8 $\alpha+$ DCs), gate 5 (LN resident CD11b + DCs), gate 6 (LN migratory CD103 DCs), and gate 7 (LN migratory CD11b + DCs). (d) In vitro cross-presentation assay using the populations as sorted under c. $(\mathbf{e}-\mathbf{g})$ Mice were injected with Flt3-L excreting B16 melanomas to expand endogenous DC pools. After 12 days, pooled lymph nodes were harvested and subjected to FACS sorting. (e) Gating strategy, in which sorted populations are gates 1 to 4 . Right panels show additional stainings on populations 1 to 3 . (f) In vitro cross-presentation assay using populations as sorted under e. Sorted cells were exposed for $5 \mathrm{~h}$ to the indicated compounds (ISCOMs

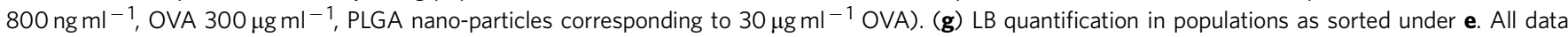
were reproduced in two independent experiments. Statistical analyses were performed using two-way ANOVA with post hoc Bonferroni tests.

the diminished LB levels, cross-presentation levels were reduced in the Igtp-1- cells, confirming the vital role for LBs in SBAinduced cross-presentation (Fig. 6e). This effect was not related to altered MHC-I or CD206 expression by DCs, or OVA processing (Supplementary Fig. 11). To validate the essential role of LBs in the observed processes, we analysed $A d r p-/-$ mice also reported to have reduced functional $\mathrm{LBs}^{31,49}$. We confirmed the lower $\mathrm{LB}$ contents, and subsequently also found reduced SBA-induced crosspresentation (Supplementary Fig. 12).

Collectively, these results show that LB formation is essential for effective SBA-induced cross-presentation in vitro.
Endosomal acidification and cytosolic antigen translocation. Enhanced endosomal antigen translocation to the cytosol has recently been proposed as a possible mechanism for SBA-induced cross-presentation $^{35}$. Still, the position of LBs relative to cytosolic translocation, and the contribution of each process to SBA-aided cross-presentation is unclear. To assay endosomal translocation, SBA-treated DCs received the mitochondrial protein cytochrome c. Extra-mitochondrial localization of cytochrome $c$ induces apoptosis, so translocation of endosomal cytochrome $\mathrm{c}$ to the cytosol will lead to reduced metabolic activity in a MTT assay. This assay, and similar experiments using the plant toxin 
saporin that inactivates ribosomes when cytosolic $^{50}$, confirmed that increased cytosolic translocation took place in the presence of SBAs (Fig. 6f). Blockade of endosomal acidification showed that SBA-induced cytosolic translocation and cross- presentation were both dependent on low endosomal $\mathrm{pH}$ (Fig. 6g; Supplementary Fig. 13a), while treatment with the proteasome inhibitor epoxomicin demonstrated that crosspresentation also critically depended on the proteasome

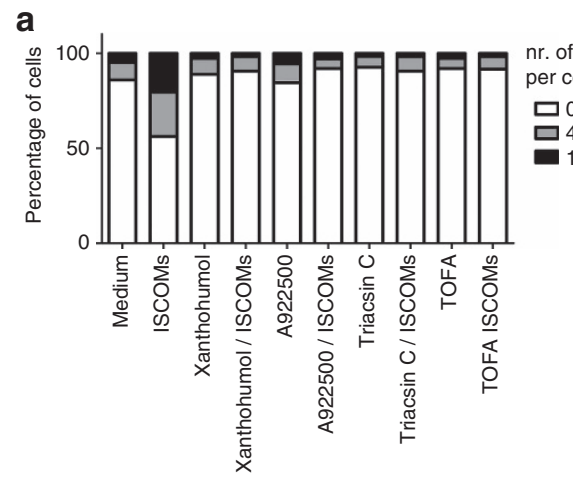

c

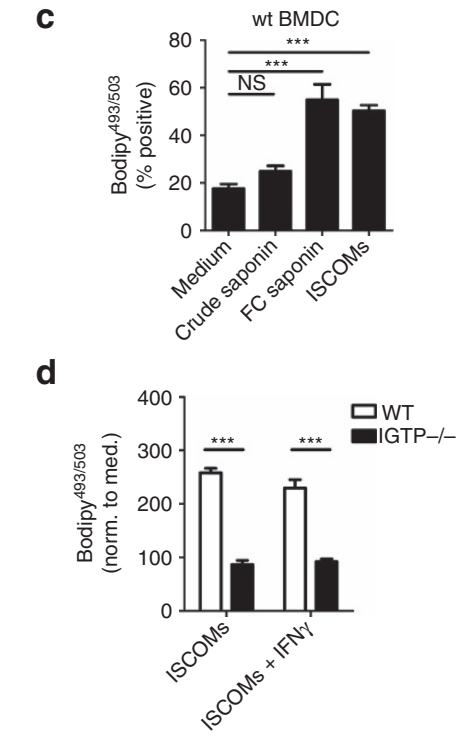

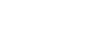
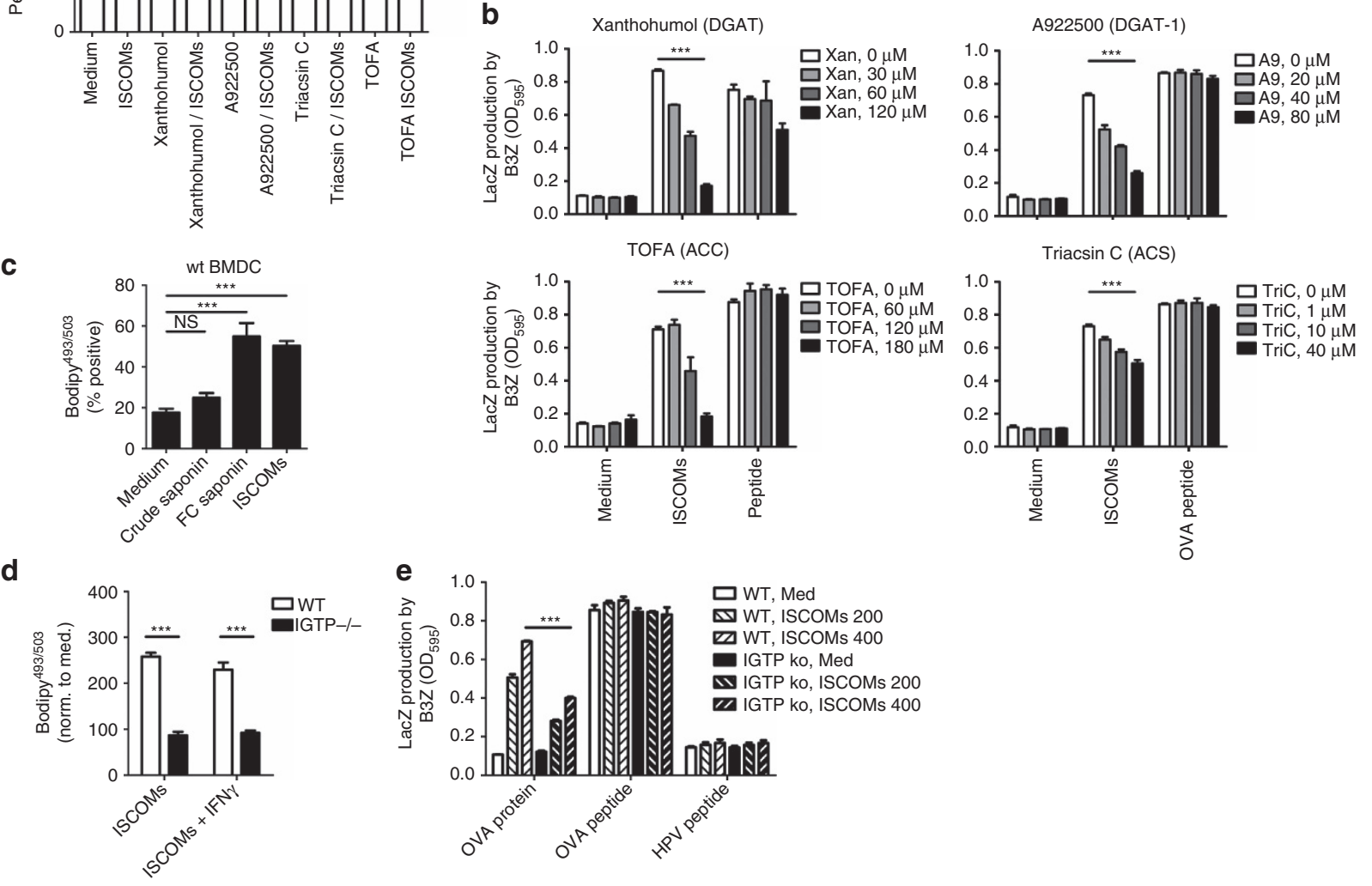

f

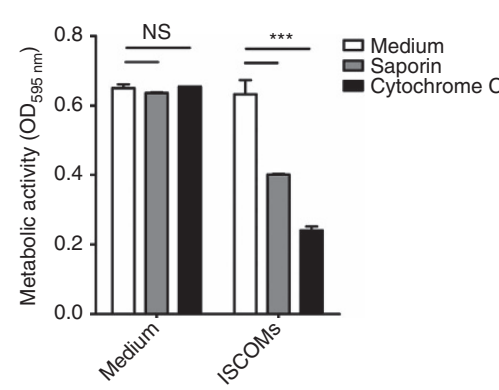

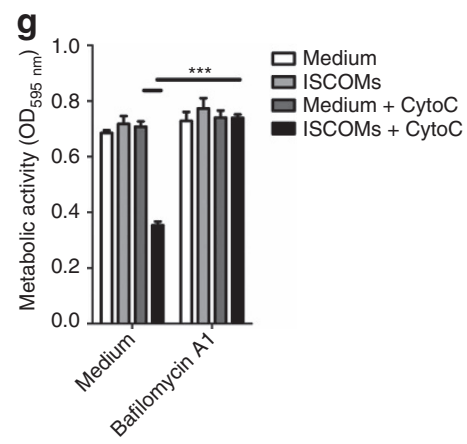

j

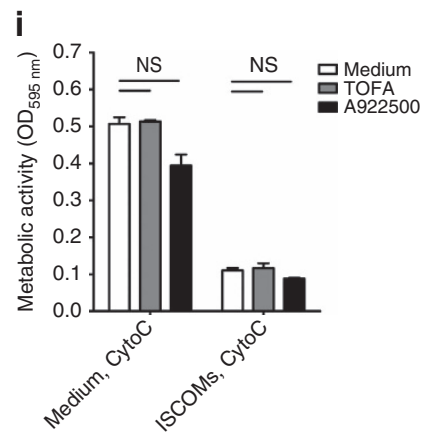

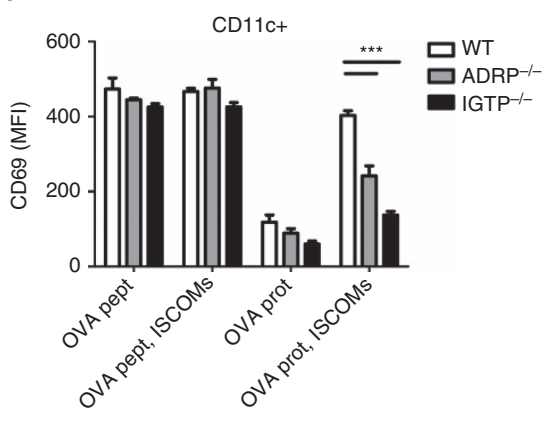

h

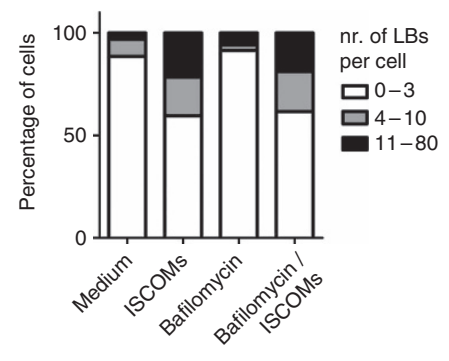

k

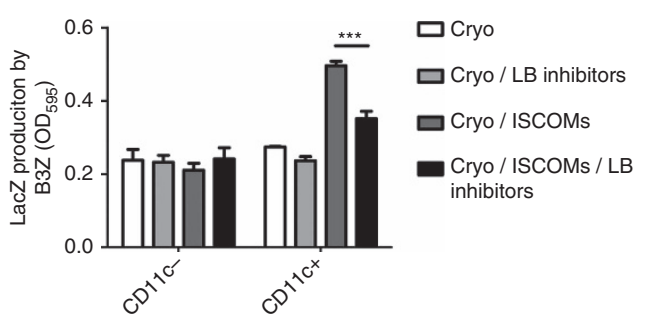


(Supplementary Fig. 13b). Strikingly, blockade of endosomal acidification (and thus endosomal escape) did not lower the numbers of SBA-induced LBs per cell (Fig. 6h). Conversely, pharmacological interference with LB induction also did not abrogate endosomal translocation (Fig. 6i).

These data show that SBA-triggered antigen translocation, but not SBA-induced LB formation, is critically preceded by endosomal acidification, and imply a role for SBA-induced LBs in facilitating the proteasomal route of cross-presentation.

LBs are vital for SBA-induced cross-presentation in vivo. To determine if cross-presentation in vivo, as induced by ISCOM vaccine injection, is LB dependent, we used similar approaches as in vitro. Cross-presentation of the ISCOMs/OVA vaccine in Adrp-/- or Igtp-/- mice lacking functional LBs was hampered compared with wild-type mice (Fig. 6j; Supplementary Fig. 14a). Consistently, injecting the DGAT inhibitors Xanthohumol or A922500 significantly reduced cross-presentation (Supplementary Fig. 14b). Importantly, OVA K ${ }^{\mathrm{b}}$ peptide presentation was intact in all cases, proving equal MHC-I levels and viability of the $\mathrm{CD} 11 \mathrm{c}+$ cells (Fig. 6j; Supplementary Fig. 14). CD11c + cell percentage or activation status of these DCs was similar in these experiments (Supplementary Fig. 15). Finally, analysis of a mix of LB inhibitors in the in situ tumour ablation model confirmed that also here the induction of LBs was essential for the SBA-induced cross-presentation (Fig. 6k).

Collectively, these results uncover a causal connection between SBA-induced LBs and cross-presentation in vivo, and link for the first time LB formation in DCs to adjuvant-mediated vaccine efficiency.

\section{Discussion}

SBAs generate strong Th1-driven cellular and humoral immune responses in various pre-clinical models and in vaccinated cancer patients ${ }^{12,37-39}$. How SBAs facilitate immune responses to vaccine antigens remains incompletely understood. We now show that SBAs specifically enhance antigen cross-presentation and the induction of LBs in the monocytic CD11b + DC subset. Using genetic and pharmacological interference, we demonstrate that LB induction in DCs is causally related to the SBAinduced increase in antigen cross-presentation and subsequent T-cell activation.

Our sorting experiments identify monocytic CD11b + DCs, as present in GM-CSF DC cultures in vitro and in LNs in vivo, as the only cells capable of reacting to SBAs by induction of cross-presentation and LB formation. Among all individual DC populations generated in in vitro Flt3-L cultures or isolated from naive spleens no SBA reactive population could be found, coinciding with the absence of migratory monocytic cells.
Interestingly, GM-CSF exposure of the Flt3 DC cultures is required for the monocytic precursors present in these cultures to respond to SBA. This finding is in line with GM-CSF (Csf-2) acting either late in differentiation or activation of monocytic cells. A previous study by Wilson et al. showing SBA-induced cross-presentation in the LN migratory DC fraction confirms our results. However, this study also reported significant levels of cross-presentation in CD $8 \alpha+$ DCs. This discrepancy with our results could possibly be explained by background crosspresentation of non-formulated OVA antigen by CD8 $\alpha+$ DCs in their study. In vivo SBA vaccination furthermore will induce inflammation, influencing co-stimulatory signals on DCs and subsequent activation of the OT-I $\mathrm{T}$ cells used in the Wilson study. Our B3Z cells react co-stimulation independent which avoids such activation biases. Earlier studies in human DCs demonstrated that SBA vaccination with the cancer antigen NY-ESO-1 induces cross-presentation by myeloid DC, such as MoDC ${ }^{51}$. Our preliminary data show LB induction in these human moDCs generated in the presence of GM-CSF, linking SBA-induced cross-presentation and LB formation also in the human context.

Thus, SBAs act to induce potent cross-presentation and LB induction in monocytic CD11b + DCs, a population of DCs distinct from the well-described $\mathrm{CD} 8 \alpha+$ cross-presenting DC. Together with the reported increase of CD11b + DCs at SBA vaccination sites, this may well explain the efficient cross-priming of CD8 + T cells by SBA vaccines.

Despite the importance of cross-presentation in health and disease, the underlying molecular processes are still poorly understood. Studies so far indicate two main pathways: the 'cytosolic' and the 'vacuolar' pathway ${ }^{52}$. Low proteolytic activity of the endocytic pathway and endosomal stress resulting in ROS production by the NADPH oxidase complex 2 (NOX2), have shown to be imperative for efficient cross-presentation of $\mathrm{CD} 8 \alpha+\mathrm{DCs}^{41}$. Our cross-presentation studies use a short time of SBA exposure ( 1 to $5 \mathrm{~h}$ ), and in this timeframe no role for ROS could be detected. Our translocation studies show that SBAs facilitate endosomal escape, and that this is essential for cross-presentation. However, since not all pore-forming saponins show adjuvant activity, and SBA activity is critically dependent on DCs, other mechanistic elements must be involved as well. Here, the presence of LBs appears to be equally essential for cross-presentation, although LBs seem to be dispensable for endosomal escape of antigens, and vice versa. The observation that both SBA-triggered processes can be uncoupled at the level of the endosome makes it unlikely that LBs alter the phagosomal environment in any direct manner, as also put forward by Bougneres et al. $^{31}$

Various types of (metabolic) stress can lead to the induction of LBs. TLR ligands were shown to increase glycolysis that fueled the

Figure 6 | SBA-induced cross-presentation is dependent on LB formation. (a) LB quantification as measured by CLSM in cells treated for $5 \mathrm{~h}$ with the indicated LB inhibitors: (Xanthohumol: $85 \mu \mathrm{M}, \mathrm{A} 922500: 40 \mu \mathrm{M}$, Triacsin C: $10 \mu \mathrm{M}$, TOFA: $120 \mu \mathrm{M}$ ). (b) In vitro cross-presentation assays in the presence of the indicated LB inhibitors (inhibited enzymes in brackets). (c,d) Flowcytometry-based analysis of LB content. Wild-type (c), or lgtp $+/+$ and lgtp $-/-$ (d) BMDCs were exposed to $800 \mathrm{ng} \mathrm{ml}^{-1}$ non-immunoactive saponin, FC saponin, ISCOMs or $250 \mathrm{ng} \mathrm{ml}^{-1} \mathrm{IFN} \gamma$ for $5 \mathrm{~h}$, washed, and stained with Bodipy ${ }^{493-503}$. (e) In vitro cross-presentation assay using IGTP knockout DCs. (f) Metabolic activity/viability (MTT) assay used to demonstrate cytosolic translocation of indicated toxins. (g) MTT assay showing endosomal acidification is needed for cytosolic translocation. Bafilomycin A1, (at $100 \mathrm{nM}$ ) is an inhibitor of endosomal v-ATPase, and thus endosomal acidification. (h) Quantification of LB induction in the presence of ISCOMs and Bafilomycin A1. (i) MTT assay showing that inhibition of LB induction does not abrogate cytosolic translocation. During the exposure to ISCOMs/Cyt C, the LB inhibitors TOFA $(120 \mu \mathrm{M})$ and A922500 $(60 \mu \mathrm{M})$ were added. (j) In vitro OT-I cross-presentation assay on isolated LN DCs from OVA protein or peptide-vaccinated wild-type, lgtp - / - , or Adrp-/ - mice. (k) B16-OVA tumour bearing mice were treated with cryo-ablation, directly followed by peritumoural injection of $30 \mu \mathrm{g}$ ISCOMs. A mixture of xanthohumol $(500 \mu \mathrm{g})$ and A922500 (150 $\mu \mathrm{g})$ was injected peritumourally, four hrs before ablation and directly after the ablation. $12 \mathrm{~h}$ later, draining LN CD11c + cells were isolated that entered the cross-presentations assays. LacZ content in B3Z cells was analysed after 2 days. All data represent means with s.e.m., except for $\mathbf{d}$ where data represent mean MFI values normalized to medium values. Similar data were obtained in two to three independent experiments. Statistical analyses were done using ANOVA with post hoc Bonferroni tests. 
de novo synthesis of fatty acids in LBs, essential for TLR ligand-induced DC activation ${ }^{53}$. Also other studies have correlated DC activation status with lipid content: When fatty acid synthesis of high-lipid-containing DCs was inhibited, these DCs lost their immunostimulatory phenotype ${ }^{54}$. Likewise, the presence of oxidized lipids in LBs was shown to hamper DC functionality and antigen presentation abilities ${ }^{55}$. This suggests that not only the absolute lipid content of LBs in DCs, but also the composition of the LBs involved can modulate immune responses. In our hands, feeding DCs fatty acids like oleic acid did induce LBs, but not cross-presentation (Supplementary Fig. 16). Finally, the presence of an established tumour (for example, before cryo-ablation) is sufficient to change the LB profile of tumour resident DCs/monocytes. This complicates a solid analysis of LB induction due to SBA administration following ablation. Together, these data imply that not all LBs are functionally equal, and that various LB types may be present at the same time, in the same cell. The fact that SBAs share the induction of LBs with certain TLR ligands places these adjuvants in a peculiar position somewhere in between the microbial and non-microbial adjuvants. Molecular analysis of the ability of SBAs to facilitate cross-presentation specifically in monocytic CD11b + DCs via endosomal escape and LB induction will be key to understand SBA adjuvant activity.

IGTP, an IFN-related GTPase associated with LBs in DCs, was shown to be important in cross-presentation of OVA coated on large latex beads ${ }^{31}$. Deletion of IGTP resulted in reduced IFN $\gamma$-induced LB numbers, but it was less established what role IFN $\gamma$ had in their cross-presentation assays. As our studies use OVA protein, which in itself is not easily cross-presented, a direct comparison with the use of latex beads is difficult, but one could speculate that the relatively large size of the beads leads to endosomal stress and possibly endosomal rupture. This putative common factor between SBA- and beads induced crosspresentation could implicate that LBs may become particularly important in forced types of cross-presentation.

The data presented here indicate an important role for IGTP and ADRP in the in vivo cross-presentation of soluble antigens after vaccination with SBAs. Whether this implicates direct involvement of these molecules, or that the effects are the indirect result of reduced LB functioning remains to be determined. To find causal connections at a more detailed level, sub-organelle/ molecular studies using purified LBs from differentially treated immune cells are required. An intriguing finding is that GM-CSF-cultured DCs and CD11b + LN DCs are uniquely able to cross-present antigen upon SBA exposure. It will be important to explore the molecular basis for this DC subset specificity, and how this can be linked to LB formation, antigen cross-presentation and immune activation.

The SBAs used in our study do not contain any known PRR ligand, and consistent with this they fail to upregulate co-stimulatory molecules on DCs in vitro (Fig. $2 \mathrm{~b}$ and ref. 17). Moreover, cross-presentation was not hampered in Tlr4-/-, or Myd88-/ - cells (Fig. 2c). Analysis of cells deficient in type I IFN or the NLRP3 inflammasome showed that these pathways were not involved either. Interestingly, others repeatedly reported an in vivo dependency of successful SBA vaccination on MyD88, while TLR4 and IL-1R seemed dispensable ${ }^{16,17}$. This suggests that effective vaccination depends on the in vivo release of endogenous danger signals or the involvement of indirect cytokine signalling. IL-18 signalling is involved in indirect NK cell and CTL activation following vaccination with $\mathrm{SBAs} \mathrm{s}^{16}$, which could partly explain the dependency on MyD88, being an adaptor for the IL18 receptor. Importantly, in this study MyD88 deficiency had no in vivo effect on maturation or vaccine antigen cross-presentation by $\mathrm{DCs}^{16}$. This research connects the initial innate cytokine- cascade with the adaptive cellular response, but leaves room for MyD88/TLR independent mechanisms of DC activation and cross-presentation with an alternative molecular basis.

Cancer vaccines evaluated to date mostly fail to change and overcome the immunosuppressive microenvironment present in many solid tumours. Current strategies using SBAs and cancer antigens (for example, NY-ESO-1) have proven to not only induce antibody responses, but also robust CD8 + T-cell expansion $^{17,37}$. Nevertheless, vaccinations with single injections of SBA-peptide compositions yielded only modest survival benefit for patients with established tumours ${ }^{56}$. Interestingly, when these vaccines were combined with TLR ligands like $\mathrm{CpG}-\mathrm{ODN}^{57,58}$, or followed up by a boosting regimen ${ }^{59}$, beneficial correlates of immunological protection could greatly be improved. We have previously shown that in situ tumour ablation can provide a source of (neo)-antigens for the generation of antitumour immune responses, provided that additional immune stimulation is given ${ }^{33}$. SBAs appeared to be superior in creating synergy between the ablation and adjuvant-induced immune effects, inducing long-lasting tumour-directed immunity ${ }^{11}$. This makes tumour ablation with local co-administration of SBAs a promising treatment strategy to explore further in clinical settings ${ }^{60}$. Future studies will have to show whether combinations of SBAs and TLR ligands are also effective combinations in ablation settings, and it will be appealing to correlate the clinical outcome to the presence and composition of LBs.

Collectively, our data shows that SBA-induced LBs are elementary in the mechanism of SBA adjuvanticity. Understanding the LB-immune connection in SBA vaccination will help to design new adjuvants for improvement of (cancer) vaccines, aiming to enhance cross-presentation and the subsequent induction of potent anti-tumour CTL responses.

\section{Methods}

Mice. Igtp - / - and Adrp - / - mice were a kind gift of S. Amigorena (Institut Curie, Paris), and have been described before ${ }^{61,62}$. Myd88-/-, Tlr4 - / - and Trif - / - mice were a kind gift of S. Akira (Osaka University, Japan). Nlrp3 - / bone-marrow was a kind gift of L. Joosten and T. Kanneganti (Nijmegen, the Netherlands). Gp91phox - / - bone-marrow was a kind gift of A. Schreiber (Berlin, Germany). All experiments using knockouts were performed with $+/+$ littermates on C57Bl/6JRccHsd background, ( $>8$ crossings). For Igtp $-/-$ and Adrp - / - mice, a common $+/+$ littermate was used (for clarity in those experiments referred to as wild type). All experiments without knockouts were performed using female wild-type C57Bl/6JRccHsd mice purchased from Charles River (Sulzfeld, Germany), and were used as $7-11$ weeks old animals. C57Bl/6 OT-IxCD90.1 ${ }^{+}$(Thy-1.1) and C57Bl/6 OT-II mice were held in the Nijmegen Animal facility. All mice were maintained on standard lab chow and sterile water, and housed under specific pathogen-free conditions in IVC cages at the Nijmegen Animal facility. All animal experiments were approved by the Animal Experimental Committee of the Radboud UMC, and were performed in accordance with institutional, national and European guidelines.

Cells. Primary cultures of bone-marrow-derived dendritic cells (BMDCs) were generated by culturing total bone marrow cells in complete medium (Roswell Park Memorial Institute (RPMI) medium (Gibco), 10\% fetal calf serum (FCS), $1 \%$ L-alanyl-L-glutamine, $0.1 \% \beta$-mercapto-ethanol and $1 \%$ antibiotics/ antimycotics (AA, Gibco)), containing $20 \mathrm{ng} \mathrm{ml}^{-1}$ granulocyte-macrophage colony stimulating factor (GM-CSF DCs, culture 7 days), or containing $200 \mathrm{ng} \mathrm{ml}^{-1}$ Fms-related tyrosine kinase 3 ligand (Flt3-L DCs, culture 10 days), as adapted from previously described protocols ${ }^{63,64}$. GM-CSF and Flt3-L were purchased from Peprotech (Rocky Hill, CT, USA). In some experiments the Flt3-L cultures were supplemented with $20 \mathrm{ng} \mathrm{ml}^{-1} \mathrm{GM}$-CSF for the indicated periods. Ex vivo DCs were isolated from pooled superficial inguinal and axillary lymph nodes, or spleens, according to previously published protocols, as indicated. For some of these experiments, mice were injected before with a B16-F10 melanoma expressing Flt3-L to expand endogenous DC pools, as described before ${ }^{34,45}$. B16-Flt3-L cells were cultured in Iscove's Modified Dulbecco's Medium (IMDM) supplemented with $5 \%$ FCS and $1 \%$ AA, and $5 \times 10^{6}$ cells were injected s.c. to establish Flt3-L-producing tumours in 9-12 days. Sorting of DC populations was performed after incubation in serum-free medium containing collagenase (Worthington, Lakewood, NJ, USA) and DNAse I (Roche) at $37^{\circ} \mathrm{C}$, later supplemented with $1 \mathrm{mM}$ EDTA. For isolation of total CD11c + fractions, a magnetic bead kit was used 
according to manufacturer's instructions (Clone N418, Miltenyi Biotech, Bergisch Gladbach, Germany). For isolation of DC subsets, total cell suspensions were sorted on a FACS Aria system (BD biosciences), based on the markers as indicated in the figures. Purity ( $>98 \%$ ) and additional markers were confirmed using a FACS Cyan system (Beckman Coulter). CD8 + T cells specific for the OVA257-264 SIINFEKL peptide in an $\mathrm{H}-2 \mathrm{~K}^{\mathrm{b}} \mathrm{MHC}-\mathrm{I}$ context, or $\mathrm{CD} 4+\mathrm{T}$ cells specific for the OVA323-339 peptide in an H-2IA ${ }^{b}$ MHC-II context, were obtained from pooled spleen and lymph nodes of OT-IxCD90.1 or OT-II transgenic mice, respectively. Before use, both cell types were purified by magnetic bead sorting (positive selection on CD8, Miltenyi). B3Z cells were cultured in Iscove's Modified Dulbecco's Medium (IMDM) supplemented with $5 \%$ FCS, $0.1 \% \beta$-mercaptoethanol, $1 \%$ antibiotics/antimycotics, $500 \mu \mathrm{g} \mathrm{ml}^{-1}$ hygromycin. The murine melanoma cell line B16F10 (ATCC) was cultured in complete medium (MEM, 5\% fetal bovine serum (Greiner Bio-one), $100 \mathrm{U} \mathrm{ml}^{-1}$ penicillin $\mathrm{G}$ sodium and $100 \mathrm{\mu g} \mathrm{ml}^{-1}$ streptomycin (Pen/Strep), MEM sodium pyruvate ( $\left.1 \mathrm{mM}\right), \mathrm{NaHCO}_{3}$, MEM vitamins, MEM non-essential amino acids (all from Gibco), $20 \mu \mathrm{M}$ betamercaptoethanol). OVA-transfected B16F10 (B16OVA, clone MO5) was cultured in complete medium supplemented with $30 \mu \mathrm{g} \mathrm{ml}^{-1}$ hygromycin and $1 \mathrm{mg} \mathrm{ml}^{-1}$ G418. B6MECsigOVA cells are transfected with murine B7.1, H-2Kb, and a construct expressing an ER targeting signal sequence, followed by the OVA257-264 CTL epitope SIINFEKL (original name B6-B7.1-sigOVA ${ }^{65}$ ). These cells were cultured in IMDM with 5\% FCS, $1 \%$ L-alanyl-L-glutamine, $0.1 \%$ $\beta$-mercapto-ethanol, $1 \%$ antibiotics/antimycotics, $100 \mu \mathrm{g} \mathrm{ml}{ }^{-1}$ Hygromycin, and $100 \mu \mathrm{g} \mathrm{ml}^{-1} \mathrm{G} 418$. All cell-lines in our study were tested mycoplasma negative.

Antibodies and reagents. For FACS experiments the following antibodies (clone name in brackets) conjugated to various fluorophores were used, together with corresponding isotypes. All antibodies were used in a 1:400 dilution in PBA, unless otherwise stated after the clone name. Anti-CD206 (C068C2; 1:100) (AbD Serotec); anti-CD8 $\alpha$ (53-6.7), anti-CD8 8.2 (53-5.8; 1:100), anti-CD11c (HL3), anti-CD69 (H1.2F3), anti-CD135 (A2F10.1; 1:100), anti-CD172 (P84), anti-Ly6C (AL21), anti-B220 (RA3-6B2; 1:100) (all BD Biosciences); anti-CD14 (Sa2-8; 1:800), anti-CD90.1-Biotin (HIS51), anti-CD115 (AFS98; 1:200), anti-I-A ${ }^{\mathrm{b}}$ / $\mathrm{I}^{\mathrm{b}} \mathrm{E}^{\mathrm{b}}$ (M5/114.15.2; 1:800), anti-SiglecH (eBio440c; 1:100) (all eBiosciences); anti-CD4 (RM4-5), anti-CD11b (M1/70), anti-CD11c (N418), anti-CD24 (M1/69), anti-CD44 (IM7; 1:1,000), anti-CD80 (16-10A1), anti-CD86 (GL1), anti-CD103 (2E7; 1:800), anti-H-2 $\mathrm{K}^{\mathrm{b}} / \mathrm{D}^{\mathrm{b}}$ (28-8-6) (all Biolegend); streptavidin-Alexa488, -555, -647 (Invitrogen; 1:800). All stainings were preceded by blocking Fc receptors using anti-CD16/CD32 antibodies (BD; 1:800). For confocal experiments the following antibodies/reagents were used: chicken anti-mouse/human ADRP (Abcam; $5 \mu \mathrm{g} \mathrm{ml}^{-1}$ ), Goat anti-chicken IgY-DyLight ${ }^{549}$ (Abcam; $5 \mu \mathrm{g} \mathrm{ml}^{-1}$ ), Bodipy ${ }^{493-503}$ dye (Invitrogen), and DAPI dye (Sigma, St Louis, MO, USA). Various assays were done in the presence of the following inhibitors or ROS scavenging compounds: diphenyleneiodonium chloride (DPI) and Bafilomycin A1 were purchased from EMD Millipore (Billerica, MA, USA); acetovanillone (Apocynin), L-ascorbic acid (Ascorbic acid), 6-hydroxy-2,5,7,8-tetramethylchromane-2-carboxylic acid (Trolox, a vitamin E analog), N-acetyl- L-cysteine (LNAC), DGAT-1 specific inhibitor A922500, chloroquine, and epoxomicin all were from Sigma; DGAT 1/2 inhibitor xanthohumol was from Tocris Biosciences (Bristol, UK); Triacsin C was from Enzo Biochem (Farmingdale, NY, USA); 5-Tetradecyloxy-2-Furoic Acid (TOFA), N-([3-(Aminomethyl)phenyl]methyl)ethanimidamide dihydrochloride $(1,400 \mathrm{~W})$, and 2-Cyano-3,12-dioxo-oleana-1,9(11)-dien-28-oic acid methyl ester, bardoxolone Methyl (CDDO-me) were from Cayman Chemical (Ann Arbor, USA); Murine IFN-gamma was from Peprotech. Water-soluble cholesterol, PMA and cis-9-octadecenoic acid (Oleic acid) were from Sigma. Ovalbumin conjugated to various alexa dyes was obtained from Invitrogen, double quenched (DQ)-OVA was purchased from ThermoFisher Scientific. Cytochrome $\mathrm{C}$ from equine hearth was purchased from Sigma. Saporin from Saponaria officinalis L (a kind gift from M. Thakur), is a type I RIP that is internalized by receptor-independent endocytosis and accumulates in late endosomes and lysosomes ${ }^{66}$. Type I RIPs and cytochrome $\mathrm{C}$ can only exhibit cytotoxicity if they can efficiently escape from the endo/lysosomes.

Adjuvants. CpG 1668 (5'-TCCATGACGTTCCTGATGCT-3') with total phosphorothioate-modified backbone was purchased from Sigma. The following non-microbial adjuvants were used (all MSD Animal Health, Boxmeer, the Netherlands): $45 / 55 \mathrm{w} / \mathrm{w}$ water-in-oil emulsion using Miglyol 840 and $0.5 \% \mathrm{w} / \mathrm{w}$ Arlacel P135; MF59 oil-in-water emulsion using squalene; Aluminum phosphate $0.75 \%$ in phosphate buffer. All adjuvants were tested in a large titration in the B3Z assays. As saponin-based adjuvants (SBAs) the Quillaja saponaria-derived adjuvants Matrix C ISCOMs (MSD) and Quil A saponin (MSD) as indicated were used. As a control, Quillaja saponaria-derived saponins not purified for immunoactive fractions were obtained from Sigma ('crude saponin'). Next to the proprietary made Matrix C, ISCOM structures were used produced via an open access method $^{40}$. In short, three immunoactive saponin fractions 'Supersap (SS)',

'Vaxsap (VX)' and 'Fraction C (FC)' were obtained from Desert King International (San Diego, US). Fifty milligram SS or FC saponin was slowly dissolved in milli-Q water to obtain $100 \mathrm{mg} \mathrm{ml}^{-1}$. Next, 1 gram $\mathrm{n}$-(D-glucityl)-n-methyldecanamide-ndecanoyl-n-met (Mega10) was dissolved in MilliQ to obtain a 20\% (w/w) solution. This solution was used to dissolve $50 \mathrm{mg}$ cholesterol (Sigma) and $50 \mathrm{mg}$ phosphatidyl choline S (Lipoid, Germany). At a temperature of $25.5^{\circ} \mathrm{C}$, the saponins were added to $1 \mathrm{ml}$ of chol/pc mixture. After adjusting the cholesterol concentration to $1 \mathrm{mg} \mathrm{ml}^{-1}$ by adding PBS, the mixture was incubated overnight in a shaker at $25.5^{\circ} \mathrm{C}$, and subsequently dialysed against PBS. ISCOMs structures of $\pm 40 \mathrm{~nm}$ were confirmed by bringing the solution onto a carbon-coated formvar plasm grid, which was activated with a $1 \%$ uranylacetate solution, and analysed using a Versa 3D electron microscope. Saponin content was analysed with an HPLC reversed phase system (Thermo RSLC 3000), using a Supersphere 60 RP select B column, and an acetonitrile/water/TFA gradient.

Flow cytometry. Phenotypic characterization of DCs and T cells was performed using standard antibody staining protocols, and analysis was done using a FACS Cyan (Beckman Coulter) or a FACS Verse system (BD). All stainings were preceded by Fc-receptor blockade using anti CD16/32 antibodies. APC maturation status was evaluated using $\mathrm{CD} 80, \mathrm{IA}^{\mathrm{b}}$ or $\mathrm{K}^{\mathrm{b}}$ staining after gating on $\mathrm{CD} 11 \mathrm{c}+$ cells. Receptor expression of CD206 (mannose receptor), $\mathrm{K}^{\mathrm{b}}$ levels, OVA uptake and degradation were used to prove equal abilities of various DCs to internalize, process and present antigens. For OVA uptake and degradation assays, cells were incubated for $5 \mathrm{~h}$ with $0.25 \mathrm{mg} \mathrm{ml}^{-1}$ OVA coupled to Alexa647 (Invitrogen) or with $1.0 \mu \mathrm{g} \mathrm{ml}^{-1}$ double quenched DQ-OVA (which fluoresces once degraded in the endolysosomal system).

B3Z and OT-I cross-presentation assays. For the cross-presentation assays, $80 \times 10^{3}$ cells (in vitro BMDCs) or $150 \times 10^{3}$ cells (ex vivo DCs) were pulsed with endotoxin-free chicken egg ovalbumin (OVA, Hyglos GmbH, Germany) in the presence of the indicated adjuvants or inhibitors. Typically, $400 \mathrm{ng} \mathrm{ml}^{-1}$ SBAs were added, and $80 \mu \mathrm{g} \mathrm{ml}^{-1}$ OVA, unless indicated otherwise. After $5 \mathrm{~h}$ of incubation at $37^{\circ} \mathrm{C}$, cells were washed and cultured for $18 \mathrm{~h}$ with $80 \times 10^{3} \mathrm{~B} 3 \mathrm{Z}$ cells. B3Z cells and positive control B6-B7.1-sigOVA cells were cultured as described elsewhere ${ }^{67}$. As a control for cell-viability and/or MHC-I expression levels, DCs were washed $30 \mathrm{~min}$ before adding the B3Z cells and pulsed with $5 \mathrm{ng} \mathrm{ml}^{-1}$ peptides. Peptides (OVA: SIINFEKL, HPV: RAHYNIVTF, Ad-E1A: SGPSNTPPEI) were obtained from J.W. Drijfhout (LUMC, Leiden, the Netherlands). In one experiment standard PEG-ylated PLGA nanoparticles formulated with OVA were used, which were produced in our lab as described elsewhere ${ }^{68}$. The amount of particles was adjusted so that the wells contained an OVA end-concentration of $30 \mu \mathrm{g} \mathrm{ml}^{-1}$. The presentation by DCs of SIINFEKL in $\mathrm{H}-2 \mathrm{~K}^{\mathrm{b}}$ results in production of $\beta$-galactosidase by the $\mathrm{B} 3 \mathrm{Z}$ cells ${ }^{65}$, which can be detected by adding $0.15 \mathrm{mM}$ chlorophenolred-h-D-galactopyranoside (Calbiochem), $9 \mathrm{mM} \mathrm{MgCl}_{2}, 0.125 \% \mathrm{NP} 40$, and $100 \mathrm{mM} \beta$-mercaptoethanol in PBS. Plates were incubated for 2 to $5 \mathrm{~h}$ at $37^{\circ} \mathrm{C}$ and absorbance values were measured using a photospectrometer $(595 \mathrm{~nm})$.

For the OT-I/II cross-presentation assays $25 \times 10^{3} \mathrm{DCs}$ were plated and treated as indicated above. Purified OT-I or OT-II cells (CD8 or CD4 MACS beads) were labelled with cell-proliferation dyes CFSE or PBSE, according to the manufacturer's instructions (Invitrogen), and $50 \times 10^{3}$ cells were incubated with the DCs for up to $72 \mathrm{~h}$. After one day of incubation, cells were stained with CD90.1, CD8, and the early activation marker CD69. After 3 days, the late stage activation marker CD44 and CFSE/PBSE dilution was analysed instead. All T-cell analyses were performed after double gating on CD8 $\beta$ and CD90.1. Cells were measured using a FACS Cyan (Beckman Coulter) or a FACS Verse system (BD).

Lipid body stainings. Lipid bodies were visualized by CLSM or FACS. For confocal microscopy, $250 \times 10^{3} \mathrm{DCs}$ were plated for $45 \mathrm{~min}$ at $37^{\circ} \mathrm{C}$ on glass coverslips coated overnight with $20 \mu \mathrm{g} \mathrm{ml}^{-1}$ fibronectin (Sigma). After washing with PBS, cells were exposed to the indicated treatment conditions in serum-free medium for $5 \mathrm{~h}$. Typically, $400 \mathrm{ng} \mathrm{ml}^{-1}$ SBAs were used, unless otherwise indicated. Next, cells were fixed in 4\% PFA solution in PBS for $15 \mathrm{~min}$. For co-staining for ADRP, fixed cells were permeabilized with $0.1 \%$ Saponin (Sigma) in PBA and incubated with primary antibodies in PBS plus $0.2 \%$ BSA/0.05\% saponin for $1 \mathrm{~h}$. Next, cells were washed and incubated with anti-chicken IgY secondary antibody conjugated to Dyelight549 (Abcam) for $45 \mathrm{~min}$. LBs were visualized by adding Bodipy ${ }^{43-503}$ probe (Invitrogen) at $7 \mu \mathrm{g} \mathrm{ml}^{-1}$ for $10 \mathrm{~min}$. Nuclear counterstaining was performed with DAPI dye according to the manufacturer's instructions. After washing, coverslips were mounted onto the slides using standard Mowiol sealing. Images were acquired using a Olympus FV1000 confocal laser scanning microscope (Olympus, Tokyo, Japan) with a $60 \times 1.35$ NA Oil immersion objective. Merged images were generated using ImageJ software (NIH, Bethesda, Maryland). For the counting of LB numbers around 15 images were taken at random spots in the sample, which were analysed using FIJI software. Nuclear defragmentation as detected by the DAPI stain was used to exclude apoptotic cells $(<2 \%)$, and only cells which were entirely visible in the image were taken along for counting. LB numbers were counted manually, yielding each time data of $90-150$ cells. For FACS analysis, cells were exposed to the indicated treatment conditions in 96 well plates for $5 \mathrm{~h}$, washed, and fixated for $10 \mathrm{~min}$ with $1 \%$ PFA. Next, cells were permeabilized using PermWash according to the manufacturer's instructions (eBioscience). Cells were then incubated with $10 \mu \mathrm{g} \mathrm{ml}^{-1}$ Bodipy ${ }^{493-503}$ for $30 \mathrm{~min}$ at $4{ }^{\circ} \mathrm{C}$. After washes, Bodipy fluorescence was detected in the FL1 (488: 530/40) channel on a FACS Cyan system (Beckman Coulter). The 
Bodipy signal integrates info from both the number, size and intensity of LBs (validated by Gocze et al. ${ }^{69}$ ).

ROS detection. The production of total cellular ROS was detected using DHR123 and H2DCFDA. $150 \times 10^{3} \mathrm{GM}$-CSF cultured BMDCs were plated in serum-free medium in 96 wells round bottom plates (Costar). Cells were pretreated for $10 \mathrm{~min}$ with 0.5 or $50 \mu \mathrm{M}$ dihydrorhodamine 123 (DHR123, Sigma), after which the following compounds were added: $400 \mathrm{ng} \mathrm{ml}^{-1}$ ISCOMs or $1 \mu \mathrm{g} \mathrm{ml}^{-1}$ phorbol 12-myristate 13-acetate (PMA, Sigma). After 15 or 45 min incubation, DHR staining was directly measured by FACS. Similarly, ROS was detected using CMH2DCFDA (Invitrogen). For this, cells were washed with HBSS containing magnesium and calcium, after which they were incubated for $45 \mathrm{~min}$ with $10 \mu \mathrm{M}$ H2DCFDA and the above stimuli dissolved in HBSS buffer.

MTT assays. GM-DCs were seeded in flat-bottom 96-well plates (Costar) at $170 \times 10^{3}$ cells per well and cultured overnight to adhere. Next, they were exposed to the indicated compounds for $5 \mathrm{~h}$ (ISCOMs: $800 \mathrm{ng} \mathrm{ml}^{-1}$, Saporin: $1 \mathrm{nM}$, Cytochrome C: $2.5 \mathrm{mg} \mathrm{ml}^{-1}$ ). After cells were washed and rested o/n, their metabolic activity/viability was measured in a standard MTT assay. In short, $10 \mu \mathrm{l}$ MTT reagent $\left(4 \mathrm{mg} \mathrm{ml}^{-1}\right)$ (Sigma) was added in medium. Plates were incubated for $1-4 \mathrm{~h}$ at $37^{\circ} \mathrm{C}$ after which supernatant was removed and $100 \mu \mathrm{l}$ lysis buffer ( $0.5 \%$ SDS, $4 \% 1 \mathrm{~N} \mathrm{HCl}$, and 3.5\% Milli-Q in isopropanol) was added. After $15 \mathrm{~min}$. of incubation, absorbance was measured at $595 \mathrm{~nm}$. Metabolic activity versus control was calculated as (treatment-blank)/(control-blank) $\times 100 \%$. Triplicate wells for each concentration were performed.

In vivo procedures. The in vivo effects of ISCOMs on cross-presentation and LB induction were analysed in wild-type, ADRP/IGTP knockouts or their $+/+$ controls $(n=6)$. For this, $300 \mu \mathrm{g}$ endofree OVA protein was injected s.c. on the femur, together with $30 \mu \mathrm{g}$ ISCOMs and/or LB inhibitors. LB inhibitors (or vehicle) were injected twice: $4 \mathrm{~h}$ before, and together with the OVA/ISCOMs injection (Xanthohumol: $500 \mu \mathrm{g}$ per injection, A922500: $150 \mu \mathrm{g}$ per injection). To control for MHC-I levels and cell viability, $40 \mu \mathrm{g}$ OVA $\mathrm{K}^{\mathrm{b}}$ peptide was injected in control mice. $12 \mathrm{~h}$ later, draining lymph nodes were harvested from which a single cell suspension was made using collagenase/DNAse containing serum-free medium. CD11c + fractions were isolated using magnetic bead sorting on CD11c according to the manufacturer's instructions. Next, these cells were processed for the B3Z/OT-I cross-presentation assays and LB stainings similarly as in the in vitro assays, with one exception: the co-incubation of DCs with B3Z cells was longer (42 instead of $18 \mathrm{~h}$ ), to allow the B3Z cells more time to produce $\beta$-galactosidase.

The cryo-ablation model, in which established tumours are destroyed by freezing has been described in more detail in the following articles ${ }^{11,33,34}$. In short, B16F10 melanomas or B16F10 cells expressing ovalbumin (B16OVA) growing s.c. on the femur with a diameter of $5-8 \mathrm{~mm}$ were treated by macroscopically freezing the entire tumour using a liquid nitrogen-cooled CryoPro Maxi device (Cortex technologies, Hadsund, Denmark). Directly before ablation, mice received an intratumoural booster shot with $20 \mu \mathrm{g}$ OVA, whenever indicated. Directly after ablation, $30 \mu \mathrm{g}$ ISCOMs or $50 \mu \mathrm{g} \mathrm{CpG}$, as indicated, was injected in the peritumoural area.

For tetramer detection of specific $\mathrm{T}$ cells in vaccinated or ablated mice, mice were injected s.c. on the right femur with $20 \mu \mathrm{g}$ endofree OVA and/or $30 \mu \mathrm{g}$ ISCOMs, or ablated as described above. Ten days later the draining lymph nodes (sup. ing.) were harvested from which a single cell suspension was made using collagenase/DNAse supplemented medium. First, cells were stained for anti $\mathrm{CD} 8 \beta .2$ on ice, after which OVA $\mathrm{K}^{\mathrm{b}}$ specific tetramers (Pelimers; Sanquin, Amsterdam, the Netherlands) were used to stain for OVA-specific T cells. Staining was done according to manufacturer's instructions, at $37^{\circ} \mathrm{C}$, and analysed on a FACS Calibur system (BD). No randomization of animals or blinding was performed in these in vivo experiments during the treatment phase, but sample analysis was performed blinded by coding samples.

Statistical analysis. Depending on the experimental layout, Student's $T$ test, oneway ANOVA, or two-way ANOVA was performed with post hoc Tukey or Bonferroni tests, as indicated in the figure legends. For in vivo data showing biological replicates sample sizes were computed using a-priori poweranalyses for ANOVA, using a power of 0.8 and alpha 0.05 . Differences were considered significant when $P$ values were smaller than 0.05 . In all figures, results are expressed as mean values from triplicates with s.e.m., unless indicated otherwise, while the following symbols were used: ${ }^{\star} P<0.05 ;{ }^{* *} P<0.01$; ${ }^{* *} P<0.001$; n.s., $P>0.05$; ND $=$ not determined.

Data availability. The data that support the findings of this study are available from the corresponding author upon request.

\section{References}

1. Kensil, C. R., Patel, U., Lennick, M. \& Marciani, D. Separation and characterization of saponins with adjuvant activity from Quillaja saponaria Molina cortex. J. Immunol. 146, 431-437 (1991).

2. Lorent, J. H., Quetin-Leclercq, J. \& Mingeot-Leclercq, M. P. The amphiphilic nature of saponins and their effects on artificial and biological membranes and potential consequences for red blood and cancer cells. Org. Biomol. Chem. 12, 8803-8822 (2014).

3. Morein, B., Sundquist, B., Hoglund, S., Dalsgaard, K. \& Osterhaus, A. Iscom, a novel structure for antigenic presentation of membrane proteins from enveloped viruses. Nature 308, 457-460 (1984).

4. Lovgren Bengtsson, K., Morein, B. \& Osterhaus, A. D. ISCOM technologybased Matrix M adjuvant: success in future vaccines relies on formulation. Expert Rev. Vaccines 10, 401-403 (2011).

5. Maraskovsky, E. et al. Development of prophylactic and therapeutic vaccines using the ISCOMATRIX adjuvant. Immunol. Cell Biol. 87, 371-376 (2009).

6. Pearse, M. J. \& Drane, D. ISCOMATRIX adjuvant: a potent inducer of humoral and cellular immune responses. Vaccine 22, 2391-2395 (2004).

7. Holzman, D. C. New data cheer champions of the danger theory. J. Natl Cancer Inst. 102, 76-78 (2010).

8. Drane, D., Gittleson, C., Boyle, J. \& Maraskovsky, E. ISCOMATRIX adjuvant for prophylactic and therapeutic vaccines. Expert Rev. Vaccines 6, 761-772 (2007).

9. McKenzie, A., Watt, M. \& Gittleson, C. ISCOMATRIX() vaccines: safety in human clinical studies. Hum. Vaccin. 6, 237-246 (2010).

10. Maraskovsky, E. et al. NY-ESO-1 protein formulated in ISCOMATRIX adjuvant is a potent anticancer vaccine inducing both humoral and CD8 + t-cell-mediated immunity and protection against NY-ESO-1 + tumors. Clin. Cancer Res. 10, 2879-2890 (2004).

11. den Brok, M. H. et al. Saponin-based adjuvants create a highly effective anti-tumor vaccine when combined with in situ tumor destruction. Vaccine 30, 737-744 (2012).

12. Nicholaou, T. et al. Regulatory T-cell-mediated attenuation of T-cell responses to the NY-ESO-1 ISCOMATRIX vaccine in patients with advanced malignant melanoma. Clin. Cancer Res. 15, 2166-2173 (2009).

13. Vantomme, V. et al. Immunologic analysis of a phase I/II study of vaccination with MAGE-3 protein combined with the AS02B adjuvant in patients with MAGE-3-positive tumors. J. Immunother. 27, 124-135 (2004).

14. Klein, O. et al. Low-dose cyclophosphamide enhances antigen-specific $\mathrm{CD} 4(+) \mathrm{T}$ cell responses to NY-ESO-1/ISCOMATRIX vaccine in patients with advanced melanoma. Cancer Immunol. Immunother. 64, 507-518 (2015).

15. Kim, Y. J. et al. Synthetic studies of complex immunostimulants from Quillaja saponaria: synthesis of the potent clinical immunoadjuvant QS-21Aapi. J. Am. Chem. Soc. 128, 11906-11915 (2006).

16. Wilson, N. S. et al. Inflammasome-dependent and -independent IL-18 production mediates immunity to the ISCOMATRIX adjuvant. J. Immunol. 192, 3259-3268 (2014).

17. Wilson, N. S. et al. ISCOMATRIX vaccines mediate CD8(+) T-cell crosspriming by a MyD88-dependent signaling pathway. Immunol. Cell Biol. 90, 540-552 (2011).

18. Joffre, O. P., Segura, E., Savina, A. \& Amigorena, S. Cross-presentation by dendritic cells. Nat. Rev. Immunol. 12, 557-569 (2012).

19. Heath, W. R. \& Carbone, F. R. Cross-presentation in viral immunity and selftolerance. Nat. Rev. Immunol. 1, 126-134 (2001).

20. Merad, M., Sathe, P., Helft, J., Miller, J. \& Mortha, A. The dendritic cell lineage: ontogeny and function of dendritic cells and their subsets in the steady state and the inflamed setting. Annu. Rev. Immunol. 31, 563-604 (2013).

21. Poulin, L. F. et al. DNGR-1 is a specific and universal marker of mouse and human Batf3-dependent dendritic cells in lymphoid and nonlymphoid tissues. Blood 119, 6052-6062 (2012).

22. Tamura, T. et al. IFN regulatory factor- 4 and -8 govern dendritic cell subset development and their functional diversity. J. Immunol. 174, 2573-2581 (2005)

23. den Haan, J. M., Lehar, S. M. \& Bevan, M. J. CD8 ( + ) but not CD8 ( - ) dendritic cells cross-prime cytotoxic T cells in vivo. J. Exp. Med. 192, 1685-1696 (2000).

24. Allan, R. S. et al. Epidermal viral immunity induced by CD8alpha + dendritic cells but not by Langerhans cells. Science 301, 1925-1928 (2003).

25. Nierkens, S., Tel, J., Janssen, E. \& Adema, G. J. Antigen cross-presentation by dendritic cell subsets: one general or all sergeants? Trends Immunol. 34, 361-370 (2013).

26. Cheong, C. et al. Microbial stimulation fully differentiates monocytes to DC-SIGN/ CD209( + ) dendritic cells for immune T cell areas. Cell 143, 416-429 (2010).

27. Farese, R. V. Jr \& Walther, T. C. Lipid droplets finally get a little R-E-S-P-E-C-T. Cell 139, 855-860 (2009).

28. Miura, S. et al. Functional conservation for lipid storage droplet association among Perilipin, ADRP, and TIP47 (PAT)-related proteins in mammals, Drosophila, and Dictyostelium. J. Biol. Chem. 277, 32253-32257 (2002).

29. Brasaemle, D. L., Dolios, G., Shapiro, L. \& Wang, R. Proteomic analysis of proteins associated with lipid droplets of basal and lipolytically stimulated 3T3-L1 adipocytes. J. Biol. Chem. 279, 46835-46842 (2004). 
30. Wan, H. C., Melo, R. C., Jin, Z., Dvorak, A. M. \& Weller, P. F. Roles and origins of leukocyte lipid bodies: proteomic and ultrastructural studies. FASEB J. 21, 167-178 (2007).

31. Bougneres, L. et al. A role for lipid bodies in the cross-presentation of phagocytosed antigens by MHC class I in dendritic cells. Immunity 31, 232-244 (2009).

32. Steinman, R. M. \& Cohn, Z. A. Identification of a novel cell type in peripheral lymphoid organs of mice. I. Morphology, quantitation, tissue distribution. J. Exp. Med. 137, 1142-1162 (1973).

33. den Brok, M. H. et al. Efficient loading of dendritic cells following cryo and radiofrequency ablation in combination with immune modulation induces antitumour immunity. Br. J. Cancer 95, 896-905 (2006).

34. Nierkens, S. et al. Immune adjuvant efficacy of CpG oligonucleotide in cancer treatment is founded specifically upon TLR9 function in plasmacytoid dendritic cells. Cancer Res. 71, 6428-6437 (2011).

35. Schnurr, M. et al. ISCOMATRIX adjuvant induces efficient cross-presentation of tumor antigen by dendritic cells via rapid cytosolic antigen delivery and processing via tripeptidyl peptidase II. J. Immunol. 182, 1253-1259 (2009).

36. Lewis, K. L. et al. Notch2 receptor signaling controls functional differentiation of dendritic cells in the spleen and intestine. Immunity 35, 780-791 (2011).

37. Duewell, P. et al. ISCOMATRIX adjuvant combines immune activation with antigen delivery to dendritic cells in vivo leading to effective cross-priming of CD8 + T cells. J. Immunol. 187, 55-63 (2011).

38. Schiott, A. et al. Posintro-HBsAg, a modified ISCOM including HBsAg, induces strong cellular and humoral responses. Int. J. Pharm. 414, 312-320 (2011).

39. Cox, R. J. et al. Evaluation of a virosomal $\mathrm{H} 5 \mathrm{~N} 1$ vaccine formulated with Matrix $\mathrm{M}$ adjuvant in a phase I clinical trial. Vaccine 29, 8049-8059 (2011).

40. Lövgren-Bengtsson, K. \& Morein, B. in Methods in Molecular Medicine Vol. 42 (ed. O’Hagan, D.T.) 239-258 (2000).

41. Kotsias, F., Hoffmann, E., Amigorena, S. \& Savina, A. Reactive oxygen species production in the phagosome: impact on antigen presentation in dendritic cells. Antioxid. Redox Signal. 18, 714-729 (2013).

42. Helft, J. et al. GM-CSF mouse bone marrow cultures comprise a heterogeneous population of $\mathrm{CD} 11 \mathrm{c}(+) \mathrm{MHCII}(+)$ macrophages and dendritic cells. Immunity 42, 1197-1211 (2015)

43. Naik, S. H. et al. Cutting edge: generation of splenic CD8 + and CD8 dendritic cell equivalents in Fms-like tyrosine kinase 3 ligand bone marrow cultures. J. Immunol. 174, 6592-6597 (2005).

44. Mayer, C. T. et al. Selective and efficient generation of functional Batf3dependent CD103 + dendritic cells from mouse bone marrow. Blood 124, 3081-3091 (2014)

45. Mach, N. et al. Differences in dendritic cells stimulated in vivo by tumors engineered to secrete granulocyte-macrophage colony-stimulating factor or Flt3-ligand. Cancer Res. 60, 3239-3246 (2000).

46. Mollah, S. A. et al. Flt3L dependence helps define an uncharacterized subset of murine cutaneous dendritic cells. J. Invest. Dermatol. 134, 1265-1275 (2014).

47. Krahmer, N., Guo, Y., Farese, R. V. Jr \& Walther, T. C. SnapShot: lipid droplets. Cell 139, 1024-1024 e1021 (2009).

48. Herber, D. L. et al. Lipid accumulation and dendritic cell dysfunction in cancer. Nat. Med. 16, 880-886 (2010).

49. Paul, A., Chang, B. H., Li, L., Yechoor, V. K. \& Chan, L. Deficiency of adipose differentiation-related protein impairs foam cell formation and protects against atherosclerosis. Circ. Res. 102, 1492-1501 (2008).

50. Weng, A. et al. Saponins modulate the intracellular trafficking of protein toxins. J. Control. Release 164, 74-86 (2012).

51. Schnurr, M. et al. Tumor antigen processing and presentation depend critically on dendritic cell type and the mode of antigen delivery. Blood 105, 2465-2472 (2005).

52. Shen, L. \& Rock, K. L. Priming of T cells by exogenous antigen cross-presented on MHC class I molecules. Curr. Opin. Immunol. 18, 85-91 (2006).

53. Everts, B. et al. TLR-driven early glycolytic reprogramming via the kinases TBK1-IKKvarepsilon supports the anabolic demands of dendritic cell activation. Nat. Immunol. 15, 323-332 (2014).

54. Ibrahim, J. et al. Dendritic cell populations with different concentrations of lipid regulate tolerance and immunity in mouse and human liver. Gastroenterology 143, 1061-1072 (2012).

55. Cao, W. et al. Oxidized lipids block antigen cross-presentation by dendritic cells in cancer. J. Immunol. 192, 2920-2931 (2014).

56. Eggermont, A. M. et al. Adjuvant ganglioside GM2-KLH/QS-21 vaccination versus observation after resection of primary tumor $>1.5 \mathrm{~mm}$ in patients with stage II melanoma: results of the EORTC 18961 randomized phase III trial. J. Clin. Oncol. 31, 3831-3837 (2013).

57. Silva, A. et al. The combination of ISCOMATRIX adjuvant and TLR agonists induces regression of established solid tumors in vivo. J. Immunol. 194, 2199-2207 (2015).

58. McCluskie, M. J. et al. CpG ODN and ISCOMATRIX adjuvant: a synergistic adjuvant combination inducing strong T-Cell IFN-gamma responses. BioMed Res. Int. 2013, 636847 (2013).
59. Chen, J. L. et al. NY-ESO-1 specific antibody and cellular responses in melanoma patients primed with NY-ESO-1 protein in ISCOMATRIX and boosted with recombinant NY-ESO-1 fowlpox virus. Int. J. Cancer 136, E590-E601 (2015).

60. van den Bijgaart, R. J. et al. Thermal and mechanical high-intensity focused ultrasound: perspectives on tumor ablation, immune effects and combination strategies. Cancer Immunol. Immunother. doi:10.1007/s00262-016-1891-9 (2016).

61. Chang, B. H. et al. Protection against fatty liver but normal adipogenesis in mice lacking adipose differentiation-related protein. Mol. Cell Biol. 26, 1063-1076 (2006).

62. Taylor, G. A. et al. Pathogen-specific loss of host resistance in mice lacking the IFN-gamma-inducible gene IGTP. Proc. Natl Acad. Sci. USA 97, 751-755 (2000).

63. Lutz, M. B. et al. An advanced culture method for generating large quantities of highly pure dendritic cells from mouse bone marrow. J. Immunol. Methods 223, 77-92 (1999).

64. Brasel, K., De Smedt, T., Smith, J. L. \& Maliszewski, C. R. Generation of murine dendritic cells from flt3-ligand-supplemented bone marrow cultures. Blood 96, 3029-3039 (2000).

65. Karttunen, J., Sanderson, S. \& Shastri, N. Detection of rare antigen-presenting cells by the lacZ T-cell activation assay suggests an expression cloning strategy for T-cell antigens. Proc. Natl Acad. Sci. USA 89, 6020-6024 (1992).

66. Gilabert-Oriol, R. et al. Reporter assay for endo/lysosomal escape of toxinbased therapeutics. Toxins 6, 1644-1666 (2014).

67. Nierkens, S. et al. In vivo colocalization of antigen and $\mathrm{CpG}$ [corrected] within dendritic cells is associated with the efficacy of cancer immunotherapy. Cancer Res. 68, 5390-5396 (2008).

68. Cruz, L. J. et al. Targeted PLGA nano- but not microparticles specifically deliver antigen to human dendritic cells via DC-SIGN in vitro. J. Control. Release 144, 118-126 (2010).

69. Gocze, P. M. \& Freeman, D. A. Factors underlying the variability of lipid droplet fluorescence in MA-10 Leydig tumor cells. Cytometry 17, 151-158 (1994).

\section{Acknowledgements}

We would like to thank E. van Dinther, J.W. Goossens, K. Horan, M. Kroesen, E. Mombarg, and R. Woestenenk for technical assistance and/or helpful discussions; L. Joosten and T. Kanneganti for kindly providing the Nlrp3 - / - bone-marrow; A. Schreiber for kindly providing the Gp91 - / - bone-marrow, the IVC department of the Nijmegen animal facility for assistance with mouse breedings; and C. Figdor and A. van Spriel for carefully reviewing this manuscript. This work was supported by grants from the Dutch Cancer Society (KUN2013-6111), the Cancer Research Institute, DFG grant TH 1810/1-1, and a Radboudumc PhD grant.

\section{Author contributions}

M.H.d.B., C.B., M.W., A.M.d.G., J.A.W. and M.M. designed and did the animal and analytical experiments; M.H.d.B., E.O.R., C.C.S., M.T., S.A. and G.J.A. conceived the project and designed the experiments; M.H.d.B., C.B. and G.J.A. wrote the paper.

\section{Additional information}

Supplementary Information accompanies this paper at http://www.nature.com/ naturecommunications

Competing financial interests: The authors declare no competing financial interests.

Reprints and permission information is available online at http://npg.nature.com/ reprintsandpermissions/

How to cite this article: den Brok, M. H. et al. Saponin-based adjuvants induce cross-presentation in dendritic cells by intracellular lipid body formation. Nat. Commun. 7, 13324 doi: 10.1038/ncomms13324 (2016).

Publisher's note: Springer Nature remains neutral with regard to jurisdictional claims in published maps and institutional affiliations.

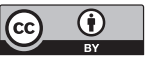

This work is licensed under a Creative Commons Attribution 4.0 International License. The images or other third party material in this article are included in the article's Creative Commons license, unless indicated otherwise in the credit line; if the material is not included under the Creative Commons license, users will need to obtain permission from the license holder to reproduce the material. To view a copy of this license, visit http://creativecommons.org/licenses/by/4.0/

(C) The Author(s) 2016 